(C) 2000 International Press

Adv. Theor. Math. Phys. 4 (2000) 335-376

\title{
Local Mirror Symmetry and
}

\section{Type IIA Monodromy of \\ Calabi-Yau manifolds}

\author{
Shinobu Hosono \\ Graduate School of Mathematical Sciences \\ University of Tokyo \\ Komaba 3-8-1, Meguro-ku \\ Tokyo 153-8914, Japan \\ hosono@ms.u-tokyo.ac.jp
}

\begin{abstract}
We propose a monodromy invariant pairing $K_{\text {hol }}(X) \otimes$ $H_{3}\left(X^{\vee}, \mathbf{Z}\right) \rightarrow \mathbf{Q}$ for a mirror pair of Calabi-Yau manifolds, $\left(X, X^{\vee}\right)$. This pairing is utilized implicitly in the previous calculations of the prepotentials for Gromov-Witten invariants. After identifying the pairing explicitly we interpret some hypergeometric series from the viewpoint of homological mirror symmetry due to Kontsevich. Also we consider the local mirror symmetry limit to del Pezzo surfaces in Calabi-Yau 3-folds.
\end{abstract}

e-print archive: http://xxx.lanl.gov/hep-th/0007071 


\section{Introduction}

In 1994, Kontsevich proposed a homological mirror symmetry [29] for a mirror pair of Calabi-Yau 3 folds. It is conjectured that there exists a categorical equivalence between the (bounded) derived category $D(X)$ of $X$ and Fukaya's $A_{\infty}$ category of Lagrangian submanifolds for the mirror $X^{\vee}$. The object of Fukaya's $A_{\infty}$ category is a (special) Lagrangian submanifold with flat $U(1)$ bundles on it and undergoes the monodromy transformations when we consider the family of manifolds $X^{\vee}$. On the other hand the object of the derived category $D(X)$ is a complex of coherent sheaves on $X$. Under the (conjectural) categorical equivalence between $D(X)$ and Fukaya's $A_{\infty}$ category the monodromy of the 3-cycles is mapped to certain automorphisms in $D(X)$, FourierMukai transformations. To write this automorphism as well as to study the equivalence of the category explicitly are important problems toward the complete understanding of the mirror symmetry.

About the possible form of the automorphisms in $D(X)$ there have been a proposal by Kontsevich [30], and his idea has been pushed forward recently by Horja [17]. They propose certain Fourier-Mukai transformations on $D(X)$ to reproduce the corresponding monodromy actions on the period integrals and compare their effects on (the D-brane charges) $H^{\text {even }}(X, \mathbf{C})$, i.e., the cohomology ring of even degrees over the coefficient $\mathbf{C}$. In this paper we remark a natural integral structure for the D-brane charges which was implicit in the previous calculations $[20],[21],[23]$. Also we will identify the generalization by Horja coincides with the local mirror symmetry in physics [31], [9] and consider the cases $\mathbf{P}^{2}$ and del Pezzo surfaces $B l_{6}, B l_{7}, B l_{8}$ in detail.

The integral structure above comes from that of the K-group $K(X)$ by the Chern character homomorphism. As for the K-group, we consider the topological $K(X)$, i.e., the set of vector bundles on $X$ divided by a certain relation (the stable equivalence). Although not all elements in $D(X)$ (, or coherent sheaves on $X$,) correspond to vector bundles on $X$, we assume all elements in $D(X)$ may be written as a complex of vector bundles and have their images in $K(X)$. Throughout this paper we write by $K(X)_{h o l}$ the image of $D(X)$ in $K(X)$ under this assumption.

Main result of this paper is a proposal ("Theorem" 2); There exists 
a monodromy invariant pairing

$$
I: K_{h o l}(X) \otimes H_{3}\left(X^{\vee}, \mathbf{Z}\right) \rightarrow \mathbf{Q}
$$

for the deformation family of Calabi-Yau manifolds $X^{\vee}$ and its mirror manifold $X$. We derive this results from Proposition 1 which has been verified explicitly for abundance of examples to establish the mirror symmetry conjecture [6]. For some special cases the mirror symmetry conjecture has been proved in [15], [32].

The homological mirror symmetry was also considered by Vafa [45] to extend the mirror symmetry conjecture [6]. In ref. [45] the yukawa coupling on the complex structure moduli space was modified to incorporate the moduli space of vector bundles on Calabi-Yau manifolds, and it was conjectured that the generalized formula enumerates open strings with boundary on a special Lagrangian 3-cycle in the mirror Calabi-Yau manifold.

The organization of this paper is as follows: In section 2, we will summarize the mirror symmetry of hypersurfaces in toric varieties following [21], [18]. In section 3 we will define type IIA monodromy and a natural integral symplectic structure implicit in the calculations summarized in section 2 . In section 4 , we will consider the monodromy associated to the local mirror symmetry to $\mathbf{P}^{2}$ and del Pezzo surfaces $B l_{6}, B l_{7}, B l_{8}$ and interpret their integral structure in language of coherent sheaves. In the final section, we will address to the recent developments in string theory which are closely related to the content of this paper.

\section{Acknowledgement}

The author would like to thank B.H. Lian and S.-T. Yau for their collaboration for the unpublished work [23], the preliminary results there are the starting point of this work. He also would like to thank H. Grosse, M. Kreuzer and S. Theisen for their kind invitation to "Duality, String Theory and M-Theory" at Ervin Srödinger Institute (March, 2000), where part of this work has been done. He also thanks A. Tsuchiya, A. Klemm, K. Yoshioka, E. Zaslow for discussions and C. Vafa for drawing his attention to the generalized mirror symmetry conjecture [45]. 
This work is supported in part by Grant-in Aid for Science Research A11740006.

\section{GKZ hypergeometric series and Prepo- tential - Review}

In this section we summarize general results about the prepotential of Calabi-Yau hypersurfaces in toric varieties obtained in [20], [21]. For simplicity we will mainly focus on hypersurfaces, however generalizations to complete intersections are straightforward (see [20] for example).

\subsection{GKZ hypergeometric series and large complex structure limit}

Let us consider a mirror pair of Calabi-Yau hypersurfaces $\left(X_{\Delta}, X_{\Delta^{*}}\right)$ described by (four dimensional) reflexive polytopes $\left(\Delta, \Delta^{*}\right)$ [2]. We denote the lattice points of the polytope $\Delta^{*}$ by $\nu_{k}^{*}(k=0, \ldots, p)$ with a convention $\nu_{0}^{*}=\overrightarrow{0}$. Then the defining equation of $X_{\Delta^{*}}$ is given by

$$
f(a)=\sum_{i=0}^{p} a_{i} X^{\nu_{i}^{*}}
$$

in terms of the torus coordinate $X_{1}, \ldots, X_{4}$ of $\left(\mathbf{C}^{*}\right)^{4} \subset \mathbf{P}_{\Sigma(\Delta)}$. The complex parameters $\left(a_{0}, \ldots, a_{p}\right) \in\left(\mathbf{C}^{*}\right)^{p+1}$ represents the deformations of the defining equation. In nice situations, to which our attention will mainly be restricted, the monomial deformations of the defining equation provide the complex structure deformations of $X_{\Delta^{*}}$. We compactify the affine space described by $a_{i}$ 's via the secondary fan so that the natural $\mathbf{C}^{*}$ actions coming from the toric ambient space $\mathbf{P}_{\Sigma(\Delta)}$ are respected. The basic object we consider in the compactification is the following lattice, so-called the lattice of relations among the vertices,

$$
L=\left\{\left(l_{0}, \ldots, l_{p}\right) \in \mathbf{Z}^{p+1} \mid \sum_{i=0}^{p} l_{i} \bar{\nu}_{i}^{*}=\overrightarrow{0}, \bar{\nu}_{i}^{*}=\left(1, \nu_{i}^{*}\right)\right\} .
$$


The secondary fan is a rational, polyhedral, complete fan in $L_{\mathbf{R}}^{\vee}$ for the dual lattice $L^{\vee}$ and its scalar extension. According to the general construction of toric varieties, the complete fan defines a toric compactification of the torus $\operatorname{Hom}_{\mathbf{Z}}\left(L, \mathbf{C}^{*}\right)$, which is our compactification of the affine space of $a_{i}$ 's. Among the cones in the secondary fan, there is a cone, called Mori cone, whose geometric meaning is the dual of the Kähler cone of $\mathbf{P}_{\Sigma\left(\Delta^{*}\right)}$. Let us denote the generators of the Mori cone $l^{(1)}, \ldots, l^{(r)}\left(r=r k\left(H^{2}\left(\mathbf{P}_{\Sigma\left(\Delta^{*}\right)}, \mathbf{Z}\right)\right)\right)$ assuming that it is simplicial for simplicity (see [21] for general non-simplicial cases). Then the Mori cone $L_{\geq 0}=\mathbf{R}_{\geq 0} l^{(1)}+\cdots+\mathbf{R}_{\geq 0} l^{(r)}$ describes the affine chart $U_{0}=$ $\operatorname{Hom}_{\text {s.g. }}\left(L_{\geq 0}, \mathbf{C}\right)$ with the coordinate $x_{k}=(-1)^{l_{0}^{(k)}} a^{l^{(k)}}(k=1, \ldots, r)$. It has been proved in general [22] that the origin $x_{1}=\cdots=x_{r}=0$ provides a large complex structure limit (LCSL) [34] and there we have only one regular period integral

$$
w_{0}(x)=\sum_{n \in \mathbf{Z}_{\geq 0}^{r}} \frac{\Gamma\left(1-\sum_{k} n_{k} l_{0}^{(k)}\right)}{\prod_{i=1}^{p} \Gamma\left(1+\sum_{k} n_{k} l_{i}^{(k)}\right)} x^{k},
$$

which corresponds to the special Lagrangian three tori cycle [44]. All other period integrals at LCSL contains logarithmic singularities. In ref.[19] such solutions are generated by classical Frobenius method. Before going into the details of the construction of the local solutions, we prepare some notations for the cohomology ring of the ambient toric variety $\mathbf{P}_{\Sigma\left(\Delta^{*}\right)}$. At a first looking, the procedure in what follows seems to be simply technical but it will turn out that there are important implications in view of homological mirror symmetry.

\subsection{Chow ring of $\mathrm{P}_{\Sigma\left(\Delta^{*}\right)}$}

Here we describe the Chow ring of the toric variety $\mathbf{P}_{\Sigma\left(\Delta^{*}\right)}$ to determine the necessary topological data of $X_{\Delta}$. The Chow ring is an abelian group generated by divisors, endowed with a commutative, graded ring structure via the intersection product. In case of toric varieties, it has a simple description in terms of the invariant divisors $D_{i}(i=0, \ldots, p)$ [39], [14]:

$$
A^{*}\left(\mathbf{P}_{\Sigma\left(\Delta^{*}\right)}\right)=\mathrm{Q}\left[D_{0}, D_{1}, \ldots, D_{p}\right] /(S R+I),
$$

where $S R$ is the Stanley-Reisner ideal for the polytope $\Delta^{*}$ and $I$ is the ideal generated by linear relations, representing rational equivalences 
among divisors;

$$
\sum_{i=0}^{p} \bar{\nu}_{i}^{*} D_{i}=\overrightarrow{0}
$$

We note here a natural non-degenerate pairing $A^{1}\left(\mathbf{P}_{\Sigma\left(\Delta^{*}\right)}\right)_{\mathbf{R}} \times L_{\mathbf{R}} \rightarrow$ $\mathbf{R}$, and thus recognize the dual cone $\left(L_{\geq 0}\right)^{\vee}$ lies in $A^{1}\left(\mathbf{P}_{\Sigma\left(\Delta^{*}\right)}\right)_{\mathbf{R}}$. In fact, according to our construction of $L_{\geq 0},\left(L_{\geq 0}\right)^{\vee}$ is the Kähler cone (or its refinement) of the ambient space $\mathbf{P}_{\Sigma\left(\Delta^{*}\right)}$. We will denote the dual basis to the generators $l^{(1)}, \ldots, l^{(r)}$ by $J_{1}, \ldots, J_{r}$. Then we may write, for example, the classical coupling $K_{a b c}^{c l}$ by

$$
K_{a b c}^{c l}:=\int_{\mathbf{P}_{\Sigma\left(\Delta^{*}\right)}}\left[X_{\Delta}\right] \cdot J_{a} \cdot J_{b} \cdot J_{c}
$$

where $\left[X_{\Delta}\right]=D_{1}+\cdots+D_{p}$ and the symbol $\int_{\mathbf{P}_{\Sigma\left(\Delta^{*}\right)}}$ means to take the coefficient of the highest degree element of (2.2) with the normalization determined by the requirement that it gives the Euler number $\chi\left(X_{\Delta}\right)$ from the top Chern class $c_{n}\left(X_{\Delta}\right)$ (see (3.59) of [21] for details). The toric part of the even cohomology $H_{\text {toric }}^{\text {even }}\left(X_{\Delta}, \mathrm{Q}\right)$ may be described by $A^{*}\left(\mathbf{P}_{\Sigma\left(\Delta^{*}\right)}\right)_{\mathbf{R}} / A n n\left(\left[X_{\Delta}\right]\right)$, where and $A n n\left(\left[X_{\Delta}\right]\right):=\{v \in$ $\left.A^{*}\left(\mathbf{P}_{\Sigma\left(\Delta^{*}\right)}\right) \mid\left[X_{\Delta}\right] v=0\right\}$.

\subsection{Prepotential and its asymptotic form}

Now we describe the local solutions about the LCSL. To this aim let us introduce in $(2.1)$ the indices $\rho_{1}, \ldots, \rho_{r}$;

$$
w_{0}(x, \rho)=\sum_{n \in \mathbf{Z}_{\geq 0}^{r}} c(n+\rho) x^{x+\rho} .
$$

The results obtained in [20] (or (3.38) of [21]) may be summarized into a concise formula using the dual bases $J_{1}, \ldots, J_{r}$ to $l^{(1)}, \ldots, l^{(r)}$ for the Kähler cone of $\mathbf{P}_{\Sigma\left(\Delta^{*}\right)}$ and restricting them to the hypersurface $X_{\Delta}$. Namely the differentials with respect to the indices in Frobenius 
method are summarized by

$$
\begin{aligned}
w_{0}\left(x, \frac{J}{2 \pi i}\right)= & w_{0}(x) \mathbf{1}+\sum_{a=1}^{r} w_{a}(x) J_{a} \\
& +\frac{1}{2 !} \sum_{a, b=1}^{r} w_{a b}(x) J_{a} J_{b}+\frac{1}{3 !} \sum_{a, b, c=1}^{r} w_{a b c}(x) J_{a} J_{b} J_{c}
\end{aligned}
$$

where products of $J_{k}$ 's are taken in the cohomology ring $H_{\text {toric }}^{\text {even }}\left(X_{\Delta}, \mathrm{Q}\right)$. This way of keeping track of the hypergeometric series was first utilized in [15] and later used in [42]. We stress here that our definition of the hypergeometric series (2.1) (or (3.38) of [21]) and that in [42] differs in the Gamma factor which normalizes the solutions. This difference in the Gamma factor is crucial to obtain integral, symplectic basis for the period integrals, although both give the solutions of PicardFuchs equation. Now to describe the formulas more concretely, let us introduce a basis $1, J_{a}, J_{b}^{(2)}, J^{(3)}$ of respective degrees by the property

$$
\left(\mathbf{1}, J^{(3)}\right)=-1,\left(J_{a}, J_{b}^{(2)}\right)=\delta_{a b},
$$

with $(A, B):=\int_{X_{\Delta}} A \wedge B$. From the reasons which will become clear soon, we shift the basis slightly to introduce a canonical "symplectic" basis of $H_{\text {toric }}^{\text {even }}\left(X_{\Delta}, \mathrm{Q}\right)$ by

$$
1, J_{a}^{(1)}:=J_{a}-\frac{c_{2}\left(X_{\Delta}\right) \wedge J_{a}}{12}, J_{b}^{(2)}, J^{(3)} .
$$

The above formal expansion of the series may be connected to the classical Frobenius method by

$$
\begin{aligned}
w_{0}\left(x, \frac{J}{2 \pi i}\right)= & w_{0}(x) \mathbf{1}+\sum_{a} D_{a}^{(1)} w_{0}(x) J_{a}^{(1)} \\
& +\sum_{b} D_{b}^{(2)} w_{0}(x) J_{b}^{(2)}+\tilde{D}^{(3)} w_{0}(x) J^{(3)}
\end{aligned}
$$

where

$$
\begin{aligned}
& D_{a}^{(1)}=\frac{1}{2 \pi i} \partial_{\rho_{a}}, \quad D_{b}^{(2)}=\frac{1}{2 !(2 \pi i)^{2}} \sum_{c, d} K_{b c d}^{c l} \partial_{\rho_{c}} \partial_{\rho_{d}} \\
& \tilde{D}^{(3)}=-\frac{1}{3 !(2 \pi i)^{3}} \sum_{a, b, c} K_{a b c}^{c l} \partial_{\rho_{a}} \partial_{\rho_{b}} \partial_{\rho_{c}}-\frac{1}{2 \pi i} \sum_{a} \frac{\left(c_{2}\left(X_{\Delta}\right), J_{a}\right)}{12} \partial_{\rho_{a}}
\end{aligned}
$$


and the notation $D_{a}^{(1)} w_{0}(x)$, for example, means an operation $\lim _{\rho \rightarrow 0} D_{a}^{(1)} w_{0}(x, \rho)$. The form of the differential operators $D^{(k)}$ using the classical yukawa coupling $K_{a b c}^{c l}=\int_{X_{\Delta}} J_{a} \wedge J_{b} \wedge J_{c}$ appeared in the references [20] (see also (3.38) of [21]) and may be reproduced directly from the formal definitions of $w_{0}\left(x, \frac{J}{2 \pi i}\right)$ and the basis (2.5). In terms of the above hypergeometric series, a closed formula for the genus zero prepotential was found to be

$$
\begin{aligned}
F(t) & =\frac{1}{2}\left(\frac{1}{w_{0}(x)}\right)^{2}\left\{w_{0}(x) \tilde{D}^{(3)} w_{0}(x)+\sum_{a} D_{a}^{(1)} w_{0}(x) D_{a}^{(2)} w_{0}(x)\right\} \\
t_{a} & =\frac{D_{a}^{(1)} w_{0}(x)}{w_{0}(x)}(a=1, \ldots, r)
\end{aligned}
$$

Especially a specific asymptotic form of the prepotential at the large radius limit was observed in [20] generalizing the results for the quintic [6], which we reproduce here in the form

$$
F(t)=\int_{X_{\Delta}} \mathcal{F}(t, J)
$$

with $\mathcal{F}(t, J)$ defined by

$$
\begin{aligned}
\mathcal{F}(t, J)= & \frac{1}{6}(t \cdot J)^{3}-\frac{c_{2}\left(X_{\Delta}\right)}{24} \wedge(t \cdot J)+\frac{\zeta(3)}{2(2 \pi i)^{3}} c_{3}\left(X_{\Delta}\right) \\
& -\frac{1}{2} \log \left(\sum_{n \in \mathbf{Z}_{\geq 0}^{r}} \frac{c\left(n+\frac{J}{2 \pi i}\right)}{c\left(\frac{J}{2 \pi i}\right)} x^{n}\right),
\end{aligned}
$$

where $t \cdot J=\sum_{a} t_{a} J_{a}$. For general proof of this formula we refer to a review article [18] (Proposition 6.7). We remark here that the prepotential was introduced to define the special Kähler geometry on the complex structure moduli space using a symplectic basis of the homology group $H_{3}\left(X_{\Delta^{*}}, \mathbf{Z}\right)$ [43]. Combined this fact with our results (2.8), we may expect that the right asymptotic form of the prepotential indicates that the hypergeometric series we have arranged about the LCSL in (2.6) is very close to the integral symplectic basis of period integrals. In the next section, we will argue that this is in fact the case relating the monodromy problem to the recent progresses on the Fourier-Mukai transformations on derived category by Horja [17]. 


\subsection{Lattice polarized K3 surfaces}

We may apply the above construction to the lattice polarized K3 surfaces [12], [28] in parallel ways. The lattice polarized K3 surfaces are defined by a lattice $M$ which has the signature $(1, r-1)$ and also admit a primitive embedding into the K3 lattice $L_{K 3}=E_{8}(-1) \oplus E_{8}(-1) \oplus H^{\oplus 3}$ where $H$ represents the rank two hyperbolic lattice. Given a lattice $M$, the lattice polarized K3 surface, $M$-polarized K3 surface, is defined to be the K3 surface whose Picard lattice is given by $M$. In a parallel way to Calabi-Yau 3 folds, we can construct mirror pair of K3 surfaces for a reflexive pair of three dimensional polytopes $\left(\Delta, \Delta^{*}\right)$, thereby we obtain the lattice polarized K3 surfaces $X_{\Delta}$ and $X_{\Delta^{*}}$ with their Picard lattices given by $M_{\Delta}$ and $M_{\Delta^{*}}$, respectively. The two lattices $M_{\Delta}$ and $M_{\Delta^{*}}$ admit primitive embedding into the $\mathrm{K} 3$ lattice and allow the inclusion

$$
L_{K 3} \supset M_{\Delta} \oplus H \oplus M_{\Delta^{*}},
$$

with finite index (see [12], [28]). The orthogonal complement of the Picard lattice gives the transcendental lattice of the K3 surface, and gives up to the factor $H$ the Picard lattice of the mirror K3 surface.

Now let us consider the prepotentials for the K3 surface $X_{\Delta}$. Since it is known that for K3 surfaces there are no instanton corrections in the prepotential due to quadratic relations (period relations) among period integrals, we may expect strict constraints on the integral basis for the period integrals of the mirror $X_{\Delta^{*}}$. Namely we should have

$$
F(t)=\frac{1}{2} \sum_{a, b} K_{a b}^{c l} t_{a} t_{b} .
$$

for the prepotential of $X_{\Delta}$. With this in mind, we arrange the series $w_{0}\left(x, \frac{J}{2 \pi i}\right)$ in $(2.6)$ by

$$
w_{0}\left(x, \frac{J}{2 \pi i}\right)=w_{0}(x) \mathbf{1}^{\prime}+\sum_{a=1}^{r} D_{a}^{(1)} w_{0}(x) J_{a}^{(1)}+\tilde{D}^{(2)} w_{0}(x) J^{(2)}
$$

where

$$
\mathbf{1}^{\prime}=\mathbf{1}-\frac{c_{2}\left(X_{\Delta}\right)}{24}, J_{a}^{(1)}=J_{a}, J^{(2)}=-\frac{c_{2}\left(X_{\Delta}\right)}{24},
$$

and

$$
D_{a}^{(1)}=\frac{1}{2 \pi i} \partial_{\rho_{a}}, \quad \tilde{D}^{(2)}=-\frac{1}{2(2 \pi i)^{2}} \sum_{a, b} K_{a b}^{c l} \partial_{\rho_{a}} \partial_{\rho_{b}}-1 .
$$


The classical couplings $K_{a b}^{c l}=\int_{X_{\Delta}} J_{a} J_{b}$ are defined for positive bases $J_{1}, \ldots, J_{r}$ of $H^{2}\left(X_{\Delta}, \mathbf{Z}\right)$. As a right generalization of the prepotential (2.7) we introduce the prepotential for K3 surfaces by

$$
F(t)=\left(\frac{1}{w_{0}(x)}\right)^{2}\left\{w_{0}(x) \tilde{D}^{(2)} w_{0}(x)+\sum_{a, b} K_{a b}^{c l} D_{a}^{(1)} w_{0}(x) D_{b}^{(1)} w_{0}(x)\right\}
$$

with the mirror map $t_{a}=\frac{D_{a}^{(1)} w_{0}(x)}{w_{0}(x)}$. Then the required asymptotics for $F(t)$ will be realized if we have the following quadratic relation,

$$
w_{0}(x) \tilde{D}^{(2)} w_{0}(x)+\frac{1}{2} \sum_{a, b} K_{a b}^{c l} D_{a}^{(1)} w_{0}(x) D_{b}^{(1)} w_{0}(x)=0
$$

which we may verify explicitly for several examples. The fact that we have the required quadratic relation for several examples already is convincing for the claim that the our arrangement (2.10) of the series provides integral and orthogonal basis for the period integrals of the transcendental cycles in $H_{2}\left(X_{\Delta^{*}}, \mathbf{Z}\right)$. Note that due to the mirror symmetry of the $\mathrm{K} 3$ surfaces the symmetric form of the transcendental lattice $H_{2}\left(X_{\Delta^{*}}, \mathbf{Z}\right)$ is given by

$$
\Sigma_{c}=\left(\begin{array}{ccc}
0 & 0 & 1 \\
0 & K_{a b}^{c l} & 0 \\
1 & 0 & 0
\end{array}\right)
$$

using the intersection form of the Picard lattice of $X_{\Delta}$. In the case of quartic hypersurfaces in $\mathbf{P}^{3}$ for example, we may verify directly the integrality of the monodromy matrices, as well as the orthogonality with respect to (2.15), through the explicit calculations of the monodromy of the hypergeometric series(2.10).

In the next sections, we will explain these phenomena in the formal expressions $(2.6)(2.10)$ from the viewpoints of the homological mirror symmetry. 


\section{Type IIA monodromy}

In this section we write the mirror pair of Calabi-Yau manifolds by $X=X_{\Delta}$ and $X^{\vee}=X_{\Delta^{*}}$ for notational simplicity. As will be evident, many of the results are not restricted to the hypersurfaces in toric varieties.

\subsection{Homological mirror symmetry and a symplec- tic basis}

The monodromy of the period integrals are symplectic with respect to the intersection form on the cycles of middle dimensions $H_{3}\left(X^{\vee}, \mathbf{Z}\right)$. The homology classes of $H_{3}\left(X^{\vee}, \mathbf{Z}\right)$ are considered as D-brane charges of (special) Lagrangian 3 cycles in $X^{\vee}$. Under the homological mirror symmetry these cycles are mapped to the elements of $D(X)$, the derived category of coherent sheaves. More precisely, Kontsevich proposes categorical equivalence between Fukaya's $A_{\infty}$ category of Lagrangian submanifolds in $X^{\vee}$ and the derived category $D(X)$ of the coherent sheaves on $X$. Therefore the skew symmetric and integral form on the homology classes $H_{3}\left(X^{\vee}, \mathbf{Z}\right)$, as D-brane charges, should have the mirror counterpart. The right notion for the D-brane charges is in the $\mathrm{K}$-group $K(X)$. Here we consider the topological $\mathrm{K}$-group and the image $K_{\text {hol }}(X)$ of $D(X)$ assuming elements in $D(X)$ may be represented by suitable complexes of vector bundles on $X$ as addressed in section 1. The skew symmetric form on $K_{\text {hol }}(X)$ may be defined by the alternating sum $\chi(\mathcal{E}, \mathcal{F})=\sum_{i}(-1)^{i} \operatorname{dim} E x t_{\mathcal{O}_{X}}^{i}(\mathcal{E}, \mathcal{F})$ for sheaves $\mathcal{E}$ and $\mathcal{F}$. If we use Riemann-Roch theorem, we may write the skew symmetric form by

$$
\chi(\mathcal{E}, \mathcal{F})=\int_{X} \operatorname{ch}\left(\mathcal{E}^{\vee} \otimes \mathcal{F}\right) \operatorname{Todd}(X) .
$$

Namely we may realize a skew symmetric form $H^{\text {even }}(X, \mathrm{Q})$ using the Chern character homomorphism ch $: K(X) \rightarrow H^{\text {even }}(X, \mathbf{Q})$. We should note that the integral structure of the image $\operatorname{ch}\left(K_{\text {hol }}(X)\right)$ is different from that of $H^{e v e n}(X, \mathbf{Z})$. This is the point we must be careful for Calabi-Yau 3-folds. (In case of K3 surfaces the latter is isomorphic to the image of $K(X)$.) 
With this background in mind, let us introduce a skew symmetric form on $H^{\text {even }}(X, \mathbf{Q})$. First we consider an involution $*$ which acts on $H^{2 i}(X, \mathbf{Q})$ by $(-1)^{i}$. Using this involution we define

$$
\begin{aligned}
\langle\alpha, \beta\rangle & =\int_{X} \alpha \wedge * \beta \wedge \operatorname{Todd}(X) \\
& =-\int_{X}\left(\alpha_{0} \beta_{6}-\alpha_{2} \beta_{4}+\alpha_{4} \beta_{2}-\alpha_{6} \beta_{0}\right) \operatorname{Todd}(X),
\end{aligned}
$$

for $\alpha, \beta \in H^{\text {even }}(X, \mathbf{Q})$. If we evaluate this skew symmetric form for the basis we have introduced in (3.4), we find that it has the 'standard' matrix form of the symplectic form, which we denote by $\Sigma_{c}$. Moreover the change of the bases

$$
J_{a}^{(1)} \rightarrow J_{a}^{(1)}+\sum_{a, b} C_{a b} J_{b}^{(2)}
$$

with symmetric form $C_{a b}=C_{b a}$ is symplectic, namely does not change the symplectic form, although it will change the integral structure introduced through the bases in $H^{\text {even }}(X, \mathbf{Q})$ (in general). We should note that this change of the bases results in the corresponding change of the asymptotic form of the prepotential (2.8) in the quadratic terms of $t$, which are known to be non-universal in contrast to other terms. Observing this fact we may summarize the results in [19], [20], [21], [23] via abundance of examples into the following statement;

Proposition 1. There exists a canonical symplectic basis of the skew symmetric form (3.2) on $H^{\text {even }}(X, \mathbf{Q})$;

$$
1, J_{a}^{S}, J_{b}^{(2)}, J^{(3)}
$$

where $J_{a}^{S}=\left(J_{a}-\sum_{b} C_{a b} J_{b}^{(2)}\right)(\operatorname{Todd}(X))^{-1}$ with some rational constants $C_{a b}=C_{b a}$. Corresponding to this basis, we have an integral symplectic basis for the period integrals about the LCSL through

$$
\begin{aligned}
w_{0}\left(x, \frac{J}{2 \pi i}\right)= & w_{0}(x) \mathbf{1}+\sum_{a} D_{a}^{(1)} w_{0}(x) J_{a}^{S} \\
& +\sum_{b} \tilde{D}_{b}^{(2)} w_{0}(x) J_{b}^{(2)}+\tilde{D}^{(3)} w_{0}(x) J^{(3)}
\end{aligned}
$$

where $\tilde{D}_{b}^{(2)}=D_{b}^{(2)}+\sum_{a} C_{a b} D_{a}^{(1)}$. Precisely the hypergeometric series appearing in the coefficients of the canonical symplectic basis of 
$H^{\text {even }}(X, \mathrm{Q})$ are integral symplectic with respect to $\Sigma_{c}$. The corresponding prepotential has the following asymptotic form:

$$
\begin{aligned}
F(t)= & \frac{1}{3 !} \sum_{a b c} K_{a b c}^{c l} t_{a} t_{b} t_{c}+\frac{1}{2} \sum_{a b} C_{a b} t_{a} t_{b} \\
& -\sum_{a} \frac{\left(c_{2}(X), J_{a}\right)}{24} t_{a}+\frac{\zeta(3)}{2(2 \pi i)^{3}} \chi(X)+\mathcal{O}(q) .
\end{aligned}
$$

In the following, we arrange the canonical symplectic basis into a row vector $\Pi^{A}(J)=\left(\mathbf{1}, J_{a}^{S}, J_{b}^{(2)}, J^{(3)}\right)$ and the corresponding period integrals in a column vector $\Pi_{B}(x)$ so that we have $w_{0}\left(x, \frac{J}{2 \pi i}\right)=$ $\Pi^{A}(J) \cdot \Pi_{B}(x)$. We should note that the formal relation (3.5) shows the homological mirror symmetry explicitly, as we may read from it the correspondences between 3 cycles in the mirror manifold $X^{\vee}$ and the elements in the derived category. The construction of the period integrals specifying suitable 3 cycles is one of the hardest part in the mirror symmetry. However our formula (3.5) replaces this problem by easier ones that thinking about coherent sheaves on Calabi-Yau manifolds. Now let us study the formula (3.5) in more details.

First of all the hypergeometric series $w_{0}(x)$ is uniquely characterized by the regularity at LCSL. It is known that this series represents the period integral for a 3 -torus $T^{3}$ coming from the complex torus $\left(\mathbf{C}^{*}\right)^{4}$ in the ambient toric variety. This cycle is proved to be special Lagrangian for the metric around the degeneration point, the LCSL point. The dual cycle to this is a vanishing cycle $S^{3}$ which appears over the principal component of the discriminants of the hypersurface $X^{\vee}$. The corresponding period integral is known to be $\tilde{D}^{(3)} w_{0}(x)$ containing the highest power of the logarithm. These cycles play an important role for the geometric mirror symmetry construction proposed in [44]. There it has been argued in general that the $T^{3}$ cycle, with flat $U(1)$ bundle on it, has its deformation space of real dimension 6 whereas the dual cycle $S^{3}$ with flat $U(1)$ bundle is rigid. Under the homological mirror symmetry these cycles should be mapped to the sheaves which have the same dimensions for their moduli spaces. A little thought tells us that they are given by the structure sheaf $\mathcal{O}_{X}$ and the skyscraper sheaf $\mathcal{O}_{p}$ for $S^{3}$ and $T^{3}$, respectively. We evaluate the Chern character for each sheaf to be $\operatorname{ch}\left(\mathcal{O}_{X}\right)=1$ and $\operatorname{ch}\left(\mathcal{O}_{p}\right)=\operatorname{Vol}_{X}$, respectively, where $\mathrm{Vol}_{X}$ is the normalized volume form of $X$. Namely we read our 
formula (3.5) as

$$
w_{0} \mathbf{1}+\cdots+\tilde{D}^{(3)} w_{0} J^{(3)}=\operatorname{ch}\left(\mathcal{O}_{X}\right) \int_{T^{3}} \Omega+\cdots+\left(-\operatorname{ch}\left(\mathcal{O}_{p}\right)\right) \int_{S^{3}} \Omega,
$$

where $\Omega$ represents the holomorphic 3-form of the mirror family. This relation indicates the following relation should hold in general;

$$
\left\langle\operatorname{ch}\left(\mathcal{E}_{\gamma}\right), w_{0}\left(x, \frac{J}{2 \pi i}\right)\right\rangle=\int_{\gamma} \Omega
$$

where we denote by $\mathcal{E}_{\gamma} \in D(X)$ the mirror image of a D-brane having the charge $\gamma \in H_{3}\left(X^{\vee}, \mathbf{Z}\right)$. As for the from

$$
J_{a}^{S}=\left(J_{a}-\sum_{b} C_{a b} J_{b}^{(2)}\right)(\operatorname{Todd}(X))^{-1},
$$

we may interprete this as the Chern character of a torsion sheaf supported on the divisor $J_{a}$. Suppose for simplicity that the divisor $D:=J_{a}$ is a smooth, irreducible subvariety in $X$. Then we may consider a coherent sheaf $\mathcal{S}$ on the subvariety $D$. We may consider this sheaf $\mathcal{S}$ in the Calabi-Yau manifold extending by zero to the outside of $D$. This is the torsion sheaf denoted by $i_{*} \mathcal{S}$, and whose Chern character may be evaluated by Riemann-Roch formula;

$$
\operatorname{ch}\left(i_{*} \mathcal{S}\right) \operatorname{Todd}(X)=i_{*}(\operatorname{ch}(\mathcal{S}) \operatorname{Todd}(D)) .
$$

If we write the components of $\operatorname{ch}\left(i_{*} \mathcal{S}\right)$, we have

$$
\begin{aligned}
\operatorname{ch}_{1}\left(i_{*} \mathcal{S}\right)= & r D \\
\operatorname{ch}_{2}\left(i_{*} \mathcal{S}\right)= & i_{*}\left(c_{1}(\mathcal{S})+\frac{r}{2} c_{1}(D)\right) \\
\operatorname{ch}_{3}\left(i_{*} \mathcal{S}\right)= & i_{*}\left(\operatorname{ch}_{2}(\mathcal{S})+\frac{1}{2} c_{1}(\mathcal{S}) c_{1}(D)+\frac{r}{12}\left(c_{1}(D)^{2}+c_{2}(D)\right)\right) \\
& -\frac{r}{12} D \cdot c_{2}(X)
\end{aligned}
$$

where $r$ is the rank of the sheaf $\mathcal{S}$ on $D$. Our formula (3.4) for $J_{a}^{S}$ is essentially this Riemann-Roch formula for the embedding of rank one sheaves. The concrete form of the base $J_{a}^{S}$ depends on the divisors, but we remark here that if a divisor $J_{a}$ describes a K3 surface[25], we may have considerable simplification for the choice of the undetermined 
constants $C_{a b}$. For example, when we considér the ideal sheaf $\mathcal{I}_{p q}$ for the torsion sheaf $\mathcal{O}_{p q}$ supported on two points on the K3 surface, we have

$$
\operatorname{ch}\left(i_{*}\left(\mathcal{I}_{p q}\right)\right)=J_{a}-\frac{1}{12} c_{2}(X) J_{a}
$$

which we evaluate from (3.8) using $c_{1}\left(\mathcal{I}_{p q}\right)=0, \operatorname{ch}_{2}\left(\mathcal{I}_{p q}\right)=-2 \mathrm{Vol}_{K 3}$ as well as $c_{1}(D)=0, c_{2}(D)=24 \mathrm{Vol}_{K 3}$. In the next section we will discuss the cases of del Pezzo surfaces after introducing certain automorphisms in the derived category $D(X)$, which was proposed by Kontsevich [30] and studied more recently by Horja [17].

\subsection{Type IIA monodromy and the monodromy in- variant pairing}

Now let us define the type IIA monodromy (group) acting on the derived category $D(X)$ by the mirror transform of the monodromy (group) acting on the 3 cycles of the mirror family. Then we understand that the actions of the monodromy on the D-brane changes, $K_{\text {hol }}(X)$, is the same as the corresponding actions on $H_{3}\left(X^{\vee}, \mathbf{Z}\right)$ under the mirror equivalence. With this definition of the monodromy we may summarize what is implied in Proposition 1 as follows;

"Theorem" 2. There exists a monodromy invariant pairing

$$
I: K_{\text {hol }}(X) \otimes H_{3}\left(X^{\vee}, \mathbf{Z}\right) \rightarrow \mathbf{Q}
$$

for the deformation family of Calabi-Yau manifolds $X^{\vee}$ and its mirror manifold $X$.

If we write the pairing $I\left(\mathcal{E}_{\gamma}, \gamma^{\prime}\right)$ for a symplectic basis $\gamma^{\prime}$ s of $H_{3}\left(X^{\vee}\right.$, Z) and its mirror transform $\mathcal{E}_{\gamma}$ 's, then our series $w_{0}\left(x, \frac{J}{2 \pi i}\right)$ should be understood by

$$
w_{0}\left(x, \frac{J}{2 \pi i}\right)=\sum_{\gamma, \gamma^{\prime}} I\left(\mathcal{E}_{\gamma}, \gamma^{\prime}\right) \operatorname{ch}\left(\mathcal{E}_{\gamma}\right) \int_{\gamma^{\prime}} \Omega
$$

where the summation is taken over a symplectic basis. Our Proposition 1 and the formula (3.6) also say that corresponding to our canonical 
symplectic basis with its symplectic form $\Sigma_{c}$, the invariant pairing has the form $\left(I\left(\mathcal{E}_{\gamma}, \gamma^{\prime}\right)\right)=\Sigma_{c}^{-1}$, identifying (3.11) with our expansion

$$
w_{0}\left(x, \frac{J}{2 \pi i}\right)=\Pi^{A}(J) \cdot \Pi_{B}(x)=: \Pi_{A}(J) \Sigma_{c}^{-1} \Pi_{B}(x),
$$

for $\Pi_{A}(J)=\left(\operatorname{ch}\left(\mathcal{E}_{\gamma}\right)\right)$ and $\Pi_{B}(x)={ }^{t}\left(\int_{\gamma} \Omega\right)$. Note that our canonical symplectic basis $\Pi^{A}(J)=\Pi_{A}(J) \Sigma_{c}^{-1}$ in Proposition 1 is still symplectic because ${ }^{t} \Sigma_{c}^{-1}=\Sigma_{c}$. The monodromy $M$ acting on the period integrals $\Pi_{B}(x) \rightarrow{ }^{t} M \Pi_{B}(x)$ should have the type IIA monodromy counterpart in the mirror, $\Pi_{A}(J) \rightarrow \Pi_{A}(J) M$. Then our identification of the invariant pairing is consistent because we have the monodromy action on $w_{0}\left(x, \frac{J}{2 \pi i}\right)=\Pi_{A}(J) \Sigma_{c}^{-1} \Pi_{B}(x) \rightarrow \Pi_{A}(J) M \Sigma_{c}^{-1 t} M \Pi_{B}(x)=$ $\Pi_{A}(J) \Sigma_{c}^{-1} \Pi_{B}(x)$ for a symplectic matrix satisfying ${ }^{t} M \Sigma_{c} M=\Sigma_{c}$.

Remark. Here we fix our convention about the monodromy actions. Let us fix a basis $\mathcal{S}=\{\gamma\}$ of $H_{3}(X, \mathbf{Z})$ and consider the Poincaré dual $\alpha_{\gamma}$ 's for the basis $H^{3}(X, \mathbf{Z})$. Then we define the skew symmetric form $\Sigma$ by

$$
\int_{\gamma} \alpha_{\gamma^{\prime}}=\int_{X} \alpha_{\gamma} \wedge \alpha_{\gamma^{\prime}}=\Sigma_{\gamma \gamma^{\prime}} .
$$

We consider the monodromy action on the basis $\left\{\alpha_{\gamma}\right\}$ by $\alpha_{\gamma} \rightarrow$ $\sum_{\gamma^{\prime}} \alpha_{\gamma^{\prime}} M_{\gamma^{\prime} \gamma}$. Then the invariance of the skew symmetric form $\Sigma$ is expressed by the condition ${ }^{t} M \Sigma M=\Sigma$, i.e., $M$ is symplectic with respect to $\Sigma$. We note that by definition of the Poincaré dual $\int_{\gamma}=\int_{X} \alpha_{\gamma} \wedge$ we have $\int_{\gamma} \rightarrow \sum_{\gamma^{\prime}} M_{\gamma^{\prime} \gamma} \int_{\gamma^{\prime}}$. The holomorphic 3-form $\Omega$ is expanded by the basis $\left\{\alpha_{\gamma}\right\}$ as follows;

$$
\Omega=\sum_{\gamma} \mathcal{G}_{\gamma} \alpha_{\gamma}=\sum_{\gamma, \gamma^{\prime}} \alpha_{\gamma}\left(\Sigma^{-1}\right)_{\gamma \gamma^{\prime}} \int_{\gamma^{\prime}} \Omega,
$$

since we have $\int_{\gamma} \Omega=\sum_{\gamma^{\prime}} \Sigma_{\gamma \gamma^{\prime}} \mathcal{G}_{\gamma^{\prime}}$. The monodromy acts on the period integral $\mathcal{G}_{\gamma}$ 's by $\mathcal{G}_{\gamma} \rightarrow \sum_{\gamma^{\prime}}\left(M^{-1}\right)_{\gamma \gamma^{\prime}} \mathcal{G}_{\gamma^{\prime}}$. We should compare the above formula with our claim (3.11) which we reproduce here,

$$
w_{0}\left(x, \frac{J}{2 \pi i}\right)=\sum_{\gamma, \gamma^{\prime}} \operatorname{ch}\left(\mathcal{E}_{\gamma}\right) I\left(\mathcal{E}_{\gamma}, \gamma^{\prime}\right) \int_{\gamma^{\prime}} \Omega .
$$

Our claim $I\left(\mathcal{E}_{\gamma}, \gamma^{\prime}\right)=\left(\Sigma^{-1}\right)_{\gamma \gamma^{\prime}}$ is consistent to identify $\Omega$ with $w_{0}\left(x, \frac{J}{2 \pi i}\right)$ under the mirror symmetry. If we define the hypergeometric series 
by $\Pi^{B}(x)=\Sigma_{c}^{-1} \Pi_{B}(x)={ }^{t}\left(\mathcal{G}_{\gamma}\right)$, then the monodromy matrix acts by $\Pi^{B}(x) \rightarrow M^{-1} \Pi^{B}(x)$. The same arguments applies to Calabi-Yau hypersurfaces (or CICYs) of arbitrary dimensions. Especially in case of even dimensions, we should replace the skew symmetric form $\Sigma$ by corresponding symmetric form, and also have the "period relations" $\int_{X} \Omega \wedge \Omega=0$

$$
\sum_{\gamma, \gamma^{\prime}} \mathcal{G}_{\gamma} \Sigma_{\gamma \gamma^{\prime}} \mathcal{G}_{\gamma^{\prime}}=0
$$

as a nontrivial consistency condition (see (2.14) for K3 surfaces).

Now we are ready to present several examples for which integral symplectic basis for the period integrals has been obtained explicitly.

Example 1 (One parameter models in [6], [27]). There are four examples of Calabi-Yau hypersurface $X_{d}(d=5,6,8,10)$ in the weighted projective spaces $\mathbf{P}(\vec{\omega})=\mathbf{P}^{4}\left(\omega_{1}, \ldots, \omega_{5}\right)$. One is the quintic in $\mathbf{P}^{4}$, whose defining data may be written $(d ; \vec{\omega})=\left(5 ; 1^{5}\right)$ specifying the degree and the weights. The others have $(d ; \vec{\omega})=\left(6 ; 2,1^{4}\right),\left(8 ; 4,1^{4}\right),\left(10 ; 5,2,1^{3}\right)$. The (even) cohomology ring has a simple form $H^{\text {even }}(X, \mathbf{Q})=\mathbf{Q}[J] /\left(J^{4}\right)$ with the normalized volume form $\operatorname{Vol}_{X}=\frac{\omega_{1} \cdots \omega_{5}}{d} J^{3}$. The topological data are given by $\left(K_{111}^{c l},\left(c_{2}, J\right), \chi\right)=(5,50,-200),(3,42,-204)$, $(2,44,-296),(1,34,-288)$, respectively for $d=5,6,8,10$. The hypergeometric series are determined by the general formula (2.1) with $l^{(1)}=(-d ; \vec{\omega})$, and we extract the hypergeometric series from the expansion

$$
\begin{aligned}
w_{0}\left(x, \frac{J}{2 \pi i}\right)= & w_{0}(x) \mathbf{1}+D^{(1)} w_{0}(x) J^{S} \\
& +\tilde{D}^{(2)} w_{0}(x) J^{(2)}+\tilde{D}^{(3)} w_{0}(x)\left(-\operatorname{Vol}_{X}\right) .
\end{aligned}
$$

If we set $J^{S}=J-\frac{c_{2} \wedge J}{12}-a J^{(2)}$ with $a=\frac{11}{2}, \frac{9}{2}, 3, \frac{1}{2}$, respectively, for $d=5,6,8,10$, we may verify that our hypergeometric series $\Pi_{B}(x)$ reproduces integral symplectic basis for period integrals constructed explicitly in refs.[6], [27]. (Precisely hypergeometric series ${ }^{t}\left(-w_{0}\right.$, $\left.-D^{(1)} w_{0}, \tilde{D}^{(2)} w_{0}, \tilde{D}^{(3)} w_{0}\right)$ coincides exactly with $N \vec{\Pi}^{\prime}=N^{t}\left(\mathcal{G}_{1}, \mathcal{G}_{2}, z^{1}\right.$ $z^{2}$ ) in ref. [27], see the formula above eq.(5.4) of [27] for the matrix $N$. The minus signs come from a difference in the overall sign of the prepotential $\mathcal{F}$.) See Example 4 for the monodromy calculations. 
Example 2 (Two parameter models in [7], [8]). The same calculation proceeds to the two parameter models $X(8) \subset \mathbf{P}(2,2,2,1,1), X(12) \subset$ $\mathbf{P}(6,2,2,1,1)$ and $X(18) \subset \mathbf{P}(9,6,1,1,1)$. For these models integral symplectic period integrals for their respective mirror $X(d)^{\vee}$ have been determined in [7], [8]. Since the necessary details may be find in the literatures, we simply reproduce here the data of their topological couplings; following the notation in [21] the non-vanishing classical yukawa couplings are summarized in

$$
\begin{gathered}
8 J_{1}^{3}+4 J_{1}^{2} J_{2} \text { for } X(8), \quad 4 J_{1}^{3}+2 J_{1}^{2} J_{2} \text { for } X(12), \\
9 J_{1}^{3}+3 J_{1}^{2} J_{2}+J_{1} J_{2}^{2} \text { for } X(18),
\end{gathered}
$$

and also the values of $c_{2} . \vec{J}:=\left(\left(c_{2}, J_{1}\right),\left(c_{2}, J_{2}\right)\right)$ are given by

$$
(56,24) \text { for } X(8), \quad(52,24) \text { for } X(12), \quad(102,36) \text { for } X(18) \text {. }
$$

Note that from these data we may reconstruct the ring $H^{\text {even }}(X, \mathbf{Q})$. For the data $\left\{l^{(1)}, l^{(2)}\right\}$ we refer to the references. What we need here is to find the constants $C_{a b}$ in the basis of Proposition 1 which reproduce the results in [7], [8]. In ref.[7] these constants are written by $\alpha, \beta, \gamma$, related to our constants $C_{11}=-\alpha, C_{12}=-\beta, C_{22}=-\gamma$. For $X(8)$ and $X(12)$, it was found that they can be arbitrary integer. Although special values $\alpha=0, \beta=-2, \gamma=0$ were chosen there, we set $\alpha=\beta=$ $\gamma=0$ in favor of our interpretation (3.9) for the K3 fibrations. Namely the $\mathrm{K} 3$ fibrations we see in the divisor $J_{2}$ simplifies the construction of our symplectic basis. In contrast to these, we need to have nonvanishing rational numbers for the $C_{a b}$ of $X(18)$. In any case, we can find the constants $C_{a b}$ which reproduce the period integrals given in [7], [8], up to the difference above, with the following choices;

$$
\begin{aligned}
X(8): J_{1}^{S} & =J_{1}-\frac{1}{12} c_{2} \wedge J_{1} \quad, \quad J_{2}^{S}=J_{2}-\frac{1}{12} c_{2} \wedge J_{2} \\
X(12): J_{1}^{S} & =J_{1}-\frac{1}{12} c_{2} \wedge J_{1} \quad, \quad J_{2}^{S}=J_{2}-\frac{1}{12} c_{2} \wedge J_{2} \\
X(18): J_{1}^{S} & =J_{1}-\frac{1}{12} c_{2} \wedge J_{1}-\frac{9}{2} J_{1}^{(2)}-\frac{3}{2} J_{2}^{(2)} \\
J_{2}^{S} & =J_{2}-\frac{1}{12} c_{2} \wedge J_{2}-\frac{3}{2} J_{2}^{(2)} .
\end{aligned}
$$

For $X(18)$, it may be convenient to define another basis $H=J_{1}, E=$ $J_{1}-3 J_{2}$ for $H^{\text {even }}(X, \mathbf{Q})$ since in this basis the classical yukawa couplings simplifies to $9 H^{3}+9 E^{3}$. This is because the divisor $E$ is isomorphic to $\mathbf{P}^{2}$ which may be contracted to a point (see the next section). 
Remark. In case of the lattice polarized K3 surfaces, there is no ambiguity in our expansion $w_{0}\left(x, \frac{J}{2 \pi i}\right)$ presented in (2.10). In this case the identifications of our bases (2.11) with suitable coherent sheaves on $X$ are

$$
\mathbf{1}^{\prime}=\operatorname{ch}\left(\mathcal{I}_{p}\right), J^{(2)}=-\operatorname{Vol}_{X}=-\operatorname{ch}\left(\mathcal{O}_{p}\right)
$$

and $J_{a}=\operatorname{ch}\left(\mathcal{O}_{D_{a}}(L)\right)$ with the torsion sheaf supported on the divisor $D_{a}=J_{a}$ tensored with a suitable line bundle $L$ on it. The line bundle $L$ on $D_{a}$ is determined requiring $\operatorname{ch}\left(\mathcal{O}_{D_{a}}(L)\right)=D_{a}-\frac{D_{a}^{2}}{2}+$ $(\operatorname{deg} L) \operatorname{Vol}_{K 3}=D_{a}$. Note that the Chern character of the ideal sheaf $\mathcal{I}_{p}$ for the skyscraper sheaf $\mathcal{O}_{p}$ may be evaluated from the exact sequence $0 \rightarrow \mathcal{I}_{p} \rightarrow \mathcal{O}_{X} \rightarrow \mathcal{O}_{p} \rightarrow 0$. Note also that the sheaf $\mathcal{I}_{p}$ is the image of $\mathcal{O}_{p}$ under the Fourier- Mukai transform $\Phi_{\mathcal{Q}} \bullet(\cdot): D(X) \rightarrow D(X)$ defined by the kernel $\mathcal{Q}$, the ideal sheaf $0 \rightarrow \mathcal{Q} \rightarrow \mathcal{O}_{X \times X} \rightarrow \mathcal{O}_{\Delta} \rightarrow 0$ for the diagonal $\Delta \subset X \times X$ [38] (cf. definitions in the next section).

The monodromy invariant pairing (3.10) in this case should be understood $I: K_{\text {hol }}(X) \otimes H_{2}\left(X^{\vee}, \mathbf{Z}\right)_{\text {trans }} \rightarrow \mathbf{Q}$, where $H_{2}\left(X^{\vee}, \mathbf{Z}\right)_{\text {trans }}$ is the transcendental lattice. For a basis of the transcendental lattice whose orthogonal form $\Sigma_{c}$ given in $(2.15)$, we have $\left(I\left(\mathcal{E}_{\gamma}, \gamma\right)\right)=\Sigma_{c}^{-1}$ and thus $w_{0}\left(x, \frac{J}{2 \pi i}\right)=\sum_{\gamma, \gamma^{\prime}} \operatorname{ch}\left(\mathcal{E}_{\gamma}\right)\left(\Sigma_{c}^{-1}\right)_{\gamma, \gamma^{\prime}} \int_{\gamma^{\prime}} \Omega$ where $\Omega$ represents the holomorphic 2 form. Full determination of the type IIA monodromy group is beyond the scope of this paper, however we may expect that the monodromy group would be generated by the $(-2)$-reflections due to Mukai on $D(X)[38]$.

The case of the elliptic curves is more simple and we may identify the type IIA monodromy with $S L(2, \mathbf{Z})$ action on $D(X)$ in [37]. This is clear but we will evaluate explicitly the monodromy of the relevant hypergeometric series in section 3.4.

\subsection{Automorphisms in derived categories}

In 1996 Kontsevich [30] considered certain automorphisms of $D(X)$ which come naturally from the homological mirror symmetry, namely Fourier-Mukai transforms with suitable choices of its kernels. To de- 
scribe them let us consider for a Calabi-Yau 3 fold $X$ the diagram:

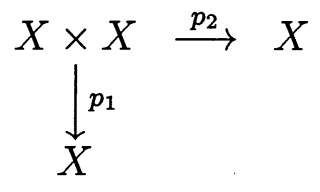

When we fix an object $\mathcal{E} \in D(X \times X)$, we may consider a functor $\Phi_{\mathcal{E}} \bullet(\cdot)=\mathbf{R}^{\bullet} p_{2_{*}}\left(\mathcal{E} \otimes^{\mathbf{L}} p_{1}^{*}(\cdot)\right): D(X) \rightarrow D(X)$. If the functors of this type define equivalences (automorphisms) of the category, they are called Fourier-Mukai transforms. We call the object $\mathcal{E} \in D(X \times X)$ in $\Phi_{\mathcal{E}}$ the kernel of the functor. The conditions on the kernel for $\Phi_{\mathcal{E}}$ to define a Furier-Mukai transform has been studied in general for smooth projective varieties in [4], [3]. The Fourier-Mukai transform $\Phi_{\mathcal{E}}: D(X) \rightarrow$ $D(X)$ induces corresponding automorphism $K_{\text {hol }}(X) \rightarrow K_{\text {hol }}(X)$ under the canonical map, and futher an automorphism on $H^{\text {even }}(X, \mathrm{Q})$ using the Chern character ring homomorphism ch : $K(X) \rightarrow H^{\text {even }}(X, \mathbf{Q})$. The identity may be realized by the torision sheaf $\mathcal{O}_{\Delta} \in D(X \times X)$ supported on the diagonal $\Delta \subset X \times X$, namely the delta function supported on the diagonal.

Now let us introduce the kernels proposed by Kontsevich. The first kernel is given by the complex,

$$
\cdots \rightarrow 0 \rightarrow \mathcal{O}_{\Delta} \otimes p_{2}^{*}(\mathcal{L}) \rightarrow 0 \rightarrow \cdots,
$$

where $\mathcal{L}$ is the sheaf of a line bundle on $X$ and the non-zero sheaf is at the zero-th position. The corresponding Furier-Mukai transform acts on a sheaf $\mathcal{F} \in D(X)$ as $\mathcal{F} \mapsto \mathcal{F} \otimes \mathcal{L}$. Since in our case of hypersurfaces in toric varieties the Picard group are generated by our basis $J_{1}, \ldots, J_{r}$ of $H^{1,1}(X, \mathbf{Z})$, we may consider $r$-independent kernels of this type. Taking the Chern character, we may summarize the Furier-Mukai transforms of this type by

$$
\mathcal{D}_{x_{k}}: \gamma \mapsto \mathrm{e}^{J_{k}} \gamma \quad\left(\gamma \in H^{\text {even }}(X, \mathbf{Q}) ; k=1, \ldots, r\right) .
$$

We have used the notation $\mathcal{D}_{x_{k}}$ because we can identify these automorphisms with the type IIA monodromy corresponding to the monodromy about the toric divisors $x_{k}=0$ at the LCSL. The second is the kernel whose cohomology is the ideal sheaf $\mathcal{I}_{\Delta}$ of the diagonal;

$$
\cdots \rightarrow 0 \rightarrow \mathcal{O}_{X \times X} \rightarrow \mathcal{O}_{\Delta} \rightarrow 0 \rightarrow \cdots,
$$


where $\mathcal{O}_{\Delta}$ at the zero-th position. The functor $\Phi_{\mathcal{E}}$ acting on a sheaf $\mathcal{F}$ results in $\cdots \rightarrow 0 \rightarrow p_{2 *}\left(\mathcal{O}_{X \times X} \otimes p_{1}^{*} \mathcal{F}\right) \rightarrow \mathcal{F} \rightarrow 0 \rightarrow \cdots$, which implies the following automorphism on $H^{\text {even }}(X, \mathbf{Q})$,

$$
\mathcal{T}_{0}: \gamma \mapsto \gamma-\left(\int_{X} \operatorname{Todd}(X) \wedge \gamma\right) \mathbf{1} \quad\left(\gamma \in H^{e v e n}(X, \mathbf{Q})\right) .
$$

Kontsevich proposed that this should reproduce the monodromy about the principal components of the discriminant of the mirror family $X^{\vee}$. Since over the principal discriminant we have vanishing cycle(s), $S^{3}$, we may identify this monodromy with the Picard-Lefschetz formula.

Recently Horja [17], generalized the kernel (3.19) to more general situation, which we may understand the local mirror symmetry counterpart of the formula (3.20). Here we briefly summarize his result to clarify its relation to the local mirror symmetry. Let us assume an exceptional locus $E$ in a Calabi-Yau hypersurface (or CICY) $X$ which may be contracted to a locus $Z$ in $X$. In the ambient toric varieties the exceptional locus $E_{0}$ of the (elementary) contractions are given by the intersections of toric divisors, $D_{i_{1}} \cap \cdots \cap D_{i_{k}}$. Here we assume our exceptional locus $E$ comes from the ambient space, namely $E_{0}$ restricted to $X$. This is exactly the local mirror symmetry considered in [26], [9]. In this setting we may consider the following diagram,

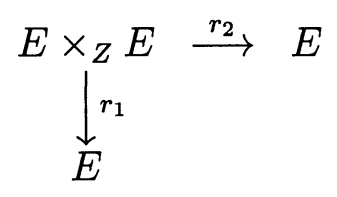

together with the embeddings $j_{a}: E \hookrightarrow X(a=1,2)$ and $j: E \times{ }_{Z} E \hookrightarrow$ $X \times X$. We write $Y:=E \times_{Z} E$. Given an object $\mathcal{I} \in D(Y)$ we may consider the functor $\Phi_{\mathcal{I}}^{\prime}(\cdot)=\mathbf{R}^{\bullet}\left(j_{2} \circ r_{2}\right)_{*}\left(\mathcal{I} \otimes^{\mathbf{L}}\left(j_{1} \circ r_{1}\right)^{*}(\cdot)\right): D(X) \rightarrow$ $D(X)$. Since we may verify $\Phi_{\mathcal{I}}^{\prime}(\cdot)=\Phi_{j_{*} \mathcal{I}}$ (Lemma 4.4 of [17]), we obtain new kernels of the Fourier-Mukai transforms in (3.16) in this way. For the local mirror symmetry version of Picard-Lefschetz formula (3.20), a naive choice of the kernel would be to consider the complex $\mathcal{I}: \cdots \rightarrow$ $0 \rightarrow \mathcal{O}_{Y} \rightarrow \mathcal{O}_{\Delta^{\prime}} \rightarrow 0 \rightarrow \cdots$, where $\Delta^{\prime} \subset Y=E \times{ }_{Z} E$ is the diagonal $\left(\Delta^{\prime} \cong E\right)$. The actual construction is more involved using the (reduced) Koszul complex for $\mathcal{O}_{\Delta^{\prime}} \cong \mathcal{O}_{E}$ and the mapping cone construction, (see ref.[17] for full details). The corresponding automorphisms on 
$H^{\text {even }}(X, \mathbf{Q})$ are determined (Proposition $4.5[17]$ ) to be

$$
\gamma \mapsto \gamma-\prod_{a=1}^{k}\left(1-\mathrm{e}^{D_{i_{a}}}\right) r_{2 *}\left(\left(j_{1} \circ r_{1}\right)^{*}(\gamma) \operatorname{Todd}(Y) r_{2}^{*}\left(\operatorname{Todd}(E)^{-1}\right)\right)
$$

for $\gamma \in H^{e v e n}(X, \mathbf{Q})$.

In our case of toric varieties, hypersurfaces (of CICYs) in toric varieties, the contraction shows itself as an extremal ray of the Mori cone (or its higher dimensional cone). In this paper we restrict our attention to the primitive contractions, where we have an extremal ray representing the contraction. Let us write the extremal ray $l^{(k)}=\left(l_{0}^{(k)} ; l_{1}^{(k)}, \ldots, l_{p}^{(k)}\right)$ in our notation prepared in section 2.1. Geometrically extremal ray represents a rational curve collapsed under the contraction. The duality of the lattice $L$ to $H^{1,1}(X, \mathbf{Z})$ (precisely $A^{1}\left(\mathbf{P}_{\Sigma\left(\Delta^{*}\right)}\right)$ ) comes from the intersection pairing between algebraic 2 cycles and the divisors. From this reason we may read off the divisor which contains the curves to be contracted from the extremal ray, namely, we simply read the negative entry of the vector $l^{(k)}$. Based on these facts we make the following definitions, $I_{+}=\left\{i \mid l_{i}^{(k)}>0\right\}, I_{-}^{\prime}=\left\{i \mid l_{i}^{(k)}<0(1 \leq i \leq p)\right\}$, $I_{-}^{\prime \prime}=\{0\}$ if $l_{0}^{(k)}<0$, and $I_{-}^{\prime \prime}=\{\phi\}$ otherwise, where $I_{-}^{\prime}$ represents the toric divisors to be contracted (the case $l_{0}^{(k)}>0$ does not occur for $\mathbf{P}_{\Sigma\left(\Delta^{*}\right)}$ being projective). We may also read the dimensions of $Z$ from the cardinality of the set $I_{+}$. Corresponding to these primitive contractions characterized by $l^{(k)}$, the automorphisms (3.22) are written more explicitly (Proposition 4.20 [17]):

$$
\mathcal{T}_{k}: \gamma \mapsto \gamma-\prod_{i \in I_{-}^{\prime}}\left(1-\mathrm{e}^{D_{i}}\right) \int_{C_{\xi}} \frac{\prod_{i \in I_{-}^{\prime \prime}}\left(1-\mathrm{e}^{D_{i}(\xi)}\right)}{\prod_{i \in I_{+}}\left(1-\mathrm{e}^{-D_{i}(\xi)}\right)} \gamma(\xi) \frac{d \xi}{2 \pi i}
$$

where $D_{i}$ represents the toric divisors represented by the vertices of $\Delta^{*}$. Note that we may write $D_{i}=\sum_{m} l_{i}^{(m)} J_{m}$ because $J_{1}, \ldots, J_{r}$ are defined to be the dual basis to $l^{(1)}, \ldots, l^{(r)}$. Then $D_{i}(\xi)$ in the above formula is defined by $D_{i}(\xi):=l_{i}^{(k)} \xi+\sum_{m \neq k} l_{i}^{(m)} J_{m}$ with a formal variable $\xi \cdot \gamma(\xi)$ is defined by the corresponding product of $D_{i}(\xi)$ 's to the representation $\gamma=\prod D_{i}$. The contour integral are understood to take all the residues about the zeros of the denominator.

In our compactification based on the secondary fan, which is briefly summarized in section 2.1, the Mori cone has a meaning to describe the 
coordinate ring of an affine chart of the compactified moduli space for the mirror family of $X^{\vee}$. In this toric compactification, the extremal ray (one dimensional cone) describes coordinate ring for one dimensional torus invariant subvariety isomorphic to $\mathbf{P}^{1}$. This is exactly the $\mathbf{P}^{1}$ whose coordinate is given by $x_{k}$ and other variables vanish. It has been proved over $\mathbf{C}$ [17] that the automorphism above coincides with the monodromy of the GKZ system around a certain loop contained in the $\mathbf{P}^{1}$. What we have now is a natural integral structure which comes from our canonical integral symplectic basis (3.4) of $H^{\text {even }}(X, \mathbf{Q})$. In fact we may verify, up to conjugation, that the automorphisms $\mathcal{D}_{x_{k}}$, $\mathcal{T}_{0}, \mathcal{T}_{k}$ on $H^{\text {even }}(X, \mathbf{Q})$ reproduce exactly the monodromy determined in [27], [6] as expected from our observation summarized in Example 1 and Example 2. Furthermore we may verify for many examples that these automorphisms are integral and symplectic with respect to our symplectic basis in Proposition 1 if we take a suitable choice for the undetermined rational constants $C_{a b}$.

\subsection{Monodromy of the elliptic curves and some other examples}

As the simplest cases of the type IIA monodromy, we present examples of the elliptic curves; $E_{6}$ : the cubic in $\mathbf{P}^{2}, E_{7}$ : the degree four curves in $\mathbf{P}^{2}(2,1,1)$ and $E_{8}$ : the degree six curves in $\mathbf{P}^{2}(3,2,1)$. For the degree $d$ curve in $\mathbf{P}^{2}(a, b, c)$ the toric data may be read from the dual polytope $\Delta^{*}(\vec{\omega})=$ Conv. $((1,0),(0,1),(-a,-b))$. For example, we determine the basis of the Mori cone to be $l=(-d ; a, b, c), \quad(d=a+b+c)$, and also $H^{\text {even }}(X, \mathbf{Q})=\mathbf{Q}[J] /\left(J^{2}\right)$ with the normalizaton $\int_{X} J=1,2,3$, respectively for $X=E_{8}, E_{7}$ and $E_{6}$. Given these data we may write the hypergeometric series:

$$
w_{0}\left(x, \frac{J}{2 \pi i}\right)=w_{0}(x)+J \frac{1}{2 \pi i} \partial_{\rho} w_{0}(x) .
$$

Since the prepotential of the elliptic curves is given simply by $F(t)=t$, there in no amibiguity to define the canomical "symplectic" basis of $H^{e v e n}(X)$. It is fixed by $\Pi^{A}(J)=\left(1,-\frac{1}{d^{\prime}} J\right)$, where $d^{\prime}=\frac{d}{a b c}$, and correspondingly integral symplectic basis for the cycles should be given by $\Pi_{B}(x)={ }^{t}\left(w_{0},-\frac{d^{\prime}}{2 \pi i} \partial_{\rho} w_{0}\right)$ accoding to our pairing. The analytic continuations of the hypergeometric series are simple exercises, for ex- 
ample, we may follow the calculation in [6] based on the Barnes integral representations. In Table 1, we list the connection matrix $N$ of the series about $x=0$ to those about $x=\infty$, from which we may determine the momodromy about $x=0$ and the discriminant $d_{i s}(x)=1-27 x, 1-64 x, 1-432 x$, repectively for $X=E_{6}, E_{7}, E_{8}$. For readers convenience we present the hypergeometric series about $x=\infty$

$$
\begin{aligned}
& w_{j}^{\infty}(x)=\sum_{k=1}^{d-1}\left(1-\alpha^{a k}\right)\left(1-\alpha^{b k}\right) \alpha^{k j} \tilde{w}_{k}(x) \\
& \tilde{w}_{k}(x)=-\frac{1}{d} \frac{1}{(2 \pi i)^{2}} \sum_{N \geq 0} \frac{\Gamma\left(a N+\frac{a k}{d}\right) \Gamma\left(b N+\frac{b k}{d}\right) \Gamma\left(N+\frac{k}{d}\right)}{\Gamma(d N+k)} x^{-N-k / 6}
\end{aligned}
$$

where $\alpha$ is the primitive root of $\alpha^{d}=1$ for $d=a+b+c$.

\begin{tabular}{|c|c|c|c|}
\hline & $E_{6}$ & $E_{7}$ & $E_{8}$ \\
\hline \hline$N$ & $\left(\begin{array}{cc}1 & 0 \\
\frac{1}{3} & -\frac{1}{3}\end{array}\right)$ & $\left(\begin{array}{cc}1 & 0 \\
\frac{1}{2} & -\frac{1}{2}\end{array}\right)$ & $\left(\begin{array}{cc}1 & 0 \\
1 & -1\end{array}\right)$ \\
\hline$M_{0}$ & $\left(\begin{array}{cc}1 & 0 \\
-3 & 1\end{array}\right)$ & $\left(\begin{array}{cc}1 & 0 \\
-2 & 1\end{array}\right)$ & $\left(\begin{array}{cc}1 & 0 \\
-1 & 1\end{array}\right)$ \\
$M_{1}$ & $\left(\begin{array}{ll}1 & 1 \\
0 & 1\end{array}\right)$ & $\left(\begin{array}{ll}1 & 1 \\
0 & 1\end{array}\right)$ & $\left(\begin{array}{ll}1 & 1 \\
0 & 1\end{array}\right)$ \\
\hline
\end{tabular}

Table 1. The connection matrix $N$ and the monodromy matrices about $x=0$ and the discriminant $d i s_{0}(x)$. The matrices $M_{0}$ and $M_{1}$ generate the modular group $\Gamma_{0}(3), \Gamma_{0}(2)$ and the modular group $\Gamma=S L(2, \mathbf{Z})$ for $E_{6}, E_{7}, E_{8}$, respectively. The connection matrix relates the hypergeometric series by ${ }^{t}\left(w_{0},-\frac{d^{\prime}}{2 \pi i} \partial_{\rho} w_{0}\right)=N^{t}\left(w_{0}^{\infty}, w_{1}^{\infty}\right)$.

As we see in the table, $\Pi_{B}(x)$ in fact provides an integral, symplectic basis for the period integrals. Furthermore it is easy to verify that the type IIA monodromy $\mathcal{T}_{0}$ and $\mathcal{D}_{x}$ exactly reproduce the the corresponding monodromy matrices when acted on the "symplectic" basis $\Pi^{A}(J)$. The modular group $\Gamma=S L(2, \mathbf{Z})$ is nothing but the modular group realized in the derived category $D(X)$ by Mukai [37].

We can extend these analysis for the lattice polarized K3 surfaces observing that there is no ambiguity in defining our canonical "orthogo- 
nal" basis for the Picard lattice. Our "Theorem" 2 (or more concretely the equation (2.10)) implies that we will obtain an orthogonal basis for the period integrals in this way.

In the rest of this section we present explicit examples of the monodromy calculations for simple cases.

Example 3 (Quartic hypersurface in $\mathrm{P}^{3}$ ). The quartic hypersurface $X(4)$ in the projective space $\mathbf{P}^{3}$ has the Picard lattice $\langle 4\rangle$, i.e., the rank one lattice with its generator $J$ of the intersection number 4 . This is a simple example of the lattice polarized $\mathrm{K} 3$ surface, and has its toric data $\Delta\left(1^{4}\right)^{*}=$ Conv. $((1,0,0),(0,1,0),(0,0,1),(-1,-1,-1))$. In Batyrev's mirror construction we may write $X(4)=X_{\Delta}$ using the dual polytope $\Delta=\Delta\left(1^{4}\right)$. The cohomology ring $H^{\text {even }}(X(4), \mathbf{Z})$ is described explicitly by $\mathbf{Z}[J] /\left(J^{3}\right)$. The hypergeometric series representing the period integral of the mirror $X(4)^{\vee}=X_{\Delta^{*}}$ may be determined by the formula (2.1) with $l=(-4 ; 1,1,1,1)$. Then following (2.10) we have

$$
w_{0}\left(x, \frac{J}{2 \pi i}\right)=w_{0}(x)\left(1-\frac{J^{2}}{4}\right)+D^{(1)} w_{0}(x) J+D^{(2)} w_{0}(x)\left(-\frac{J^{2}}{4}\right) .
$$

We identify this expansion by $w_{0}\left(x, \frac{J}{2 \pi i}\right)=\Pi_{A}(J) \cdot \Pi^{B}(x)$ introducing the notations $\Pi_{A}(J)=\left(1-\frac{J^{2}}{4}, J,-\frac{J^{2}}{4}\right)$, and $\Pi^{B}(x)={ }^{t}\left(w_{0}(x)\right.$, $\left.D^{(1)} w_{0}(x), \tilde{D}^{(2)} w_{0}(x)\right)$. The way of identification is slightly differs from that in Proposition 1 for 3 folds cases, since we verify right intersection form for the basis $\Pi_{A}(J)$ (see below). More explicitly we have

$$
w_{0}(x), \quad D^{(1)} w_{0}(x)=\partial_{\rho} w_{0}(x), \quad \tilde{D}^{(2)} w_{0}(x)=-2 \partial_{\rho}^{2} w_{0}(x)-w_{0}(x)
$$

for the period integral about $x=0$. The analytic continuation to $x=\infty$ is similar to the cases in elliptic curves. Since it is easy to determine the monodromy about $x=0$ and $x=\infty$, we can determine the monodromy group once we know the connection formula of the two local solutions. In Appendix, we briefly summarize the monodromy calculation done in [6] applying to the present case. (The calculations are essentially the same for all monodromy calculations done in this paper.) In any case we obtain the monodromy matrices $M_{0}, M_{\infty}$ and, in turn, $M_{0}$ and $M_{1}=\left(M_{0} M_{\infty}\right)^{-1}$ after determining the connection matrix $N\left(\right.$ where $\Pi^{B}(x)=N \Pi_{\infty}^{B}(x)$ with $\Pi_{\infty}^{B}(x)={ }^{t}\left(w_{0}^{\infty}, w_{1}^{\infty}, w_{2}^{\infty}\right)$, see 
Appendix for the definition $w_{j}^{\infty}(x)$, ):

$$
M_{0}=\left(\begin{array}{ccc}
1 & 0 & 0 \\
1 & 1 & 0 \\
-2 & -4 & 1
\end{array}\right), M_{1}=\left(\begin{array}{lll}
0 & 0 & 1 \\
0 & 1 & 0 \\
1 & 0 & 0
\end{array}\right), N=\left(\begin{array}{ccc}
1 & 0 & 0 \\
-\frac{1}{4} & \frac{1}{2} & \frac{1}{4} \\
-\frac{1}{2} & -\frac{1}{2} & 0
\end{array}\right)
$$

Now it is an easy exercise to verify the same monodromy matrices $M_{0}$ and $M_{1}$ from the Fourier-Mukai transformations (3.18) and (3.20), respectively, in the basis $\Pi_{A}(J)$. (Precisely we should have $\Pi_{A}(J) \rightarrow$ $\Pi_{A}(J) M^{-1}$ if $\Pi^{B}(x) \rightarrow M \Pi^{B}(x)$ according to Remark after (3.11). However this is simply a matter of the identification of the monodromy actions, i.e., either we reverse the monodromy actions on $\Pi^{B}(x)$ or reverse the Fourier-Mukai transformations (3.18), (3.20).) Also we may verify that the monodromy matrices $M_{0}$ and $M_{1}$ are orthogonal with respect to the canonical orthogonal form $\Sigma_{c}$ in (2.15). We should note that the orthogonal form (2.15) is nothing but the symmetric form $\langle\alpha, \beta\rangle=-\int_{X}\left(\alpha_{0} \beta_{4}-\alpha_{2} \beta_{2}\right) \operatorname{Todd}(X)$ (3.2) expressed in the basis $\{1-$ $\left.\frac{J^{2}}{4}, J,-\frac{J^{2}}{4}\right\}$. Corresponding period integrals $\Pi^{B}(x)$ are identified with the period integrals of the transcendental cycles of the mirror $X(4)^{\vee}$. As a consistency we verify explicitly the period relation (3.12), or explicitly using (2.14),

$$
w_{0}(x) D^{(2)} w_{0}(x)+\frac{4}{2}\left(D^{(1)} w_{0}(x)\right)^{2}=0
$$

The quadratic relation above may be used to determine the modular property of the mirror map $x=x(q)$ defined by $t=\frac{D^{(1)} w_{0}(x)}{w_{0}(x)}$ with $q=\mathrm{e}^{2 \pi i t}$. Namely the monodromy $M_{0}$ simply acts on $t$ by $t \rightarrow t+1$, while the monodromy $M_{1}$ acts

$$
t=\frac{D^{(1)} w_{0}(x)}{w_{0}(x)} \rightarrow \frac{D^{(1)} w_{0}(x)}{D^{(2)} w_{0}(x)}=\frac{w_{0}(x) D^{(1)} w_{0}(x)}{w_{0}(x) D^{(2)} w_{0}(x)}=-\frac{1}{2 t} .
$$

Now we recall that the modular group $\Gamma_{0}(N)_{+}$is a normalizer of $\Gamma_{0}(N)$ in $S L(2, \mathbf{R})$, defined by adding the Fricke involution $t \rightarrow-\frac{1}{N t}$. Since for $N=2$ we may write $\Gamma_{0}(2)+=\left\langle\left(\begin{array}{cc}0 & -1 \\ 2 & 0\end{array}\right),\left(\begin{array}{ll}1 & 1 \\ 0 & 1\end{array}\right)\right\rangle$, we identify the modular group of our mirror map $x=x(q)$ with $\Gamma_{0}(2)_{+}$. In this way we may arrive at the known result in [33]. 
Example 4 (One parameter models in [6], [27]). For readers convenience we list the monodromy matrices for the one parameter models in Example 1. Although the results are obtained in [6], [27], we reproduce them here for the comparison with the type IIA monodromy calculations. The monodromy calculations for the hypergeometric series $\Pi_{B}(x)={ }^{t}\left(w_{0}, D^{(1)} w_{0}, \tilde{D}^{(2)} w_{0}, \tilde{D}^{(3)} w_{0}\right)$ are exactly the same as in Example 3. We define the hypergeometric series about $x=\infty$ by

$$
\begin{aligned}
w_{j}^{\infty}(x) & =\sum_{k=1}^{d}\left(\prod_{i=1}^{4}\left(1-\alpha^{\omega_{i} k}\right)\right) \alpha^{k j} \tilde{w}_{k}(x)(j=0,1, \ldots, d-1), \\
\tilde{w}_{k}(x) & =-\frac{1}{d} \frac{1}{(2 \pi i)^{4}} \sum_{N=0}^{\infty} \frac{\prod_{i=1}^{5} \Gamma\left(\omega_{i}\left(N+\frac{k}{d}\right)\right)}{\Gamma(d N+k)} x^{-N-\frac{k}{d}}
\end{aligned}
$$

All of these series are not linearly independent but have some relations, for example,$w_{0}^{\infty}+\cdots+w_{d-1}^{\infty}=0$ (see [27]). Taking an independent set we define $\Pi_{B}^{\infty}(x)={ }^{t}\left(w_{0}^{\infty}(x), w_{1}^{\infty}(x), w_{2}^{\infty}(x), w_{d-1}^{\infty}(x)\right)$ and the connection matrix $N$ by the relation $\Pi_{B}(x)=N \Pi_{B}^{\infty}(x)$. Since the monodromy about $x=\infty$ is simple in the basis $\Pi_{B}^{\infty}(x)$, we can determine the corresponding action on $\Pi_{B}(x)$ using the connection matrix. The results are listed in Table 2.

We leave for reader's exercise to verify the same monodromy matrices follow from the type IIA monodromy, namely Fourier-Mukai transformations (3.18)(3.20) acting on our canonical symplectic basis $\Pi^{A}(J)=\left(1, J-\frac{c_{2} \wedge J}{12}-a J^{(2)}, J^{(2)},-\operatorname{Vol}_{X}\right)$ with $a=\frac{11}{2}, \frac{9}{2}, 3, \frac{1}{2}$, respectively for $d=5,6,8,10$ (see Example 1). The action (3.20) reproduces the monodromy about the discriminant, which is in the form of PicardLefshetz fromula for all $X_{d}$;

$$
M_{1}=\left(M_{0} M_{\infty}\right)^{-1}=\left(\begin{array}{cccc}
1 & 0 & 0 & 1 \\
0 & 1 & 0 & 0 \\
0 & 0 & 1 & 0 \\
0 & 0 & 0 & 1
\end{array}\right)
$$




\begin{tabular}{|c|c|c|c|c|c|c|c|c|c|c|c|c|}
\hline$d$ & \multicolumn{4}{|c|}{$M_{0}$} & \multicolumn{4}{|c|}{$M_{\infty}$} & \multicolumn{4}{|c|}{$N$} \\
\hline \multirow{4}{*}{5} & 11 & 0 & 0 & $0)$ & 1 & 0 & & $-1\rangle$ & 1 & 0 & 0 & 0 \\
\hline & 1 & 1 & 0 & 0 & -1 & 1 & 0 & 1 & $-\frac{2}{5}$ & $\frac{2}{5}$ & $\frac{1}{r}$ & $-\frac{1}{\varepsilon}$ \\
\hline & 8 & 5 & 1 & 0 & -3 & -5 & 1 & 3 & $-\frac{21}{5}$ & $\frac{5}{1}$ & $\frac{3}{5}$ & $-\frac{8}{2}$ \\
\hline & -5 & 3 & -1 & 1) & 5 & -8 & 1 & $-4)$ & 1 & -1 & 0 & $0^{\circ}$ \\
\hline & 1 & 0 & 0 & $0)$ & 1 & 0 & 0 & $-1\rangle$ & 1 & 0 & 0 & 0 \\
\hline & 1 & 1 & 0 & 0 & -1 & 1 & 0 & 1 & $-\frac{1}{3}$ & $\frac{1}{3}$ & $\frac{1}{3}$ & $-\frac{1}{3}$ \\
\hline 6 & 6 & 3 & 1 & 0 & -3 & -3 & 1 & 3 & -3 & 0 & 1 & -2 \\
\hline & -4 & 3 & -1 & 1) & 4 & -6 & 1 & $-3)$ & 1 & -1 & 0 & 0 \\
\hline & /1 & 0 & 0 & $0)$ & 1 & 0 & 0 & $-1)$ & 1 & 0 & 0 & 0 \\
\hline 8 & 1 & 1 & 0 & 0 & -1 & 1 & 0 & 1 & $-\frac{1}{2}$ & $\frac{1}{2}$ & $\frac{1}{2}$ & $-\frac{1}{2}$ \\
\hline 8 & 4 & 2 & 1 & 0 & -2 & -2 & 1 & 2 & -3 & 0 & 1 & -2 \\
\hline & -4 & 2 & -1 & $1)$ & 4 & -4 & 1 & $-3)$ & 1 & -1 & 0 & 0 \\
\hline & 1 & 0 & 0 & $0\rangle$ & 1 & 0 & 0 & $-1)$ & 1 & 0 & 0 & 0 \\
\hline 10 & 1 & 1 & 0 & 0 & -1 & 1 & 0 & 1 & 0 & 0 & 1 & -1 \\
\hline 10 & 1 & 1 & 1 & 0 & 0 & -1 & 1 & 0 & -1 & -1 & 0 & -1 \\
\hline & -3 & 0 & -1 & 1) & 3 & -1 & 1 & $-2)$ & 1 & -1 & 0 & 0 \\
\hline
\end{tabular}

Table 2. The connection matrix $N$ and the monodromy matrices for one parameter models $X_{d}(d=5,6,8,10)$. Matrices are written in terms of our canonical symplectic basis defiend by $w_{0}\left(x, \frac{J}{2 \pi i}\right)=\Pi^{A}(J) . \Pi_{B}(x)$.

\section{Local limit of the monodromy}

\subsection{Calabi-Yau models}

Here we briefly summarize some properties of elliptic Calabi-Yau 3-folds over the Hirzebruch surface $\mathbf{F}_{1}$. These Calabi-Yau 3-folds are studied extensively in the context of F-theory [35], [36], [26]. We will study in detail the local mirror symmetry limit of their monodromy.

Let us consider the affine space $\mathbf{C}^{7}$ with its coordinate $x_{1}, x_{2}, \ldots, x_{7}$ and the torus actions generated by

$$
\left(\mathbf{C}^{*}\right)_{(a, b, c)}^{3}=\left\langle\begin{array}{c}
(a, b, c, 0,0,0,0)_{\lambda}, \\
(0,0,-1,1,1,0,-1)_{\mu}, \\
(0,0,-2,0,0,1,1)_{\nu}
\end{array}\right\rangle
$$


where $\lambda, \mu, \nu \neq 0$ and $\left(c_{1}, c_{2}, \ldots, c_{7}\right)_{\lambda}$ represents the $\mathbf{C}^{*}$ action $x_{i} \mapsto$ $\lambda^{c_{i}} x_{i}(\lambda \neq 0)$. The quotient of $\mathbf{C}^{7}$ by this group does not behave well, however if we remove some bad loci we can obtain a good manifold [10]. In our case, the bad loci are determined by the toric data coming from the reflexive polytope and there are two different ways to make sense of the quotient by removing either the loci

$$
\mathcal{Z}=\left(x_{1}=x_{2}=x_{3}=0\right) \cup\left(x_{4}=x_{5}=0\right) \cup\left(x_{6}=x_{7}=0\right) \subset \mathbf{C}^{7},
$$

or

$$
\begin{aligned}
\tilde{\mathcal{Z}}=\left(x_{1}=x_{2}=x_{4}=x_{5}=0\right) \cup & \left(x_{3}=x_{7}=0\right) \\
& \cup\left(x_{4}=x_{5}=x_{6}=0\right) \subset \mathbf{C}^{7} .
\end{aligned}
$$

Correspondingly we have toric varieties;

$$
\mathbf{P}_{\Sigma(a, b, c)}:=\left(\mathbf{C}^{7} \backslash \mathcal{Z}\right) /\left(\mathbf{C}^{*}\right)_{(a, b, c)}^{3}, \tilde{\mathbf{P}}_{\Sigma(a, b, c)}:=\left(\mathbf{C}^{7} \backslash \tilde{\mathcal{Z}}\right) /\left(\mathbf{C}^{*}\right)_{(a, b, c)}^{3}
$$

Looking into the detail of the relevant toric data, it is easy to see that these two toric varieties are related by the flop in two dimensions (see e.g., [35], [26]). In the both cases the general section $s$ of the anti- canonical bundle defines a Calabi-Yau hypersurface $X=s^{-1}(0)$. In what follows we will be mainly concerned with the hypersurface in $\tilde{\mathbf{P}}_{\Sigma(a, b, c)}$ because it contains a del Pezzo surface as an toric divisor, which may be contracted. (The hypersurface in $\mathbf{P}_{\Sigma(a, b, c)}$ contains a rational elliptic surface and K3 surface, and will be studied elsewhere.) We denote the defining equation of the Calabi-Yau hypersurfaces by

$$
\begin{aligned}
& \tilde{X}_{E(8)}: x_{1}^{2}+x_{2}^{3}+x_{3}^{6}\left\{\left(x_{4}^{6}+x_{5}^{6}\right) x_{6}^{12}+\left(x_{4}^{18}+x_{5}^{18}\right) x_{7}^{12}\right\}=0 \subset \tilde{\mathbf{P}}_{\Sigma(3,2,1)}, \\
& \tilde{X}_{E(7)}: x_{1}^{2}+x_{2}^{4}+x_{3}^{4}\left\{\left(x_{4}^{4}+x_{5}^{4}\right) x_{6}^{8}+\left(x_{4}^{12}+x_{5}^{12}\right) x_{7}^{8}\right\}=0 \subset \tilde{\mathbf{P}}_{\Sigma(2,1,1)}, \\
& \tilde{X}_{E(6)}: x_{1}^{3}+x_{2}^{3}+x_{3}^{3}\left\{\left(x_{4}^{3}+x_{5}^{3}\right) x_{6}^{6}+\left(x_{4}^{9}+x_{5}^{9}\right) x_{7}^{6}\right\}=0 \subset \tilde{\mathbf{P}}_{\Sigma(1,1,1)},
\end{aligned}
$$

where it is understood that all possible deformations, which are homogeneous polynomials with respect to the torus actions $\mathbf{C}^{*}(a, b, c)$, are included in the defining equations. The homogeneous degree has been determined from the anti-canonical bundle (divisor) $-K_{\mathbf{P}_{\Sigma}}$ of the ambient spaces. We see explicitly from the defining equation that the divisor $D_{7}=\left(x_{7}=0\right)$ restricted to $\tilde{X}_{E(k)}$ describes the del Pezzo surfaces $B l_{k}$ for $k=6,7,8$. Also the divisor $D_{3}=\left(x_{3}=0\right)$ restricted 
to $\tilde{X}_{E(k)}$ is $\mathbf{P}^{2}$. (Hereafter we will abuse the notation $D_{i}$ to represent the corresponding divisor restricted to the hypersurface $\tilde{X}_{E(k)}$.) In our situation the del Pezzo surfaces appear as the degree $d=a+b+c$ hypersurface in $\mathbf{P}^{3}(a, b, c, 1)$.

Our Calabi-Yau hypersurfaces are typical examples of those by Batyrev, and we may associate a pair of reflexive polytopes for mirror symmetry of these spaces. For example for the Calabi-Yau hypersurfaces $\tilde{X}_{E(k)}(k=6,7,8)$ we associate a pair of reflexive polytopes $\left(\Delta(a, b, c), \Delta^{*}(a, b, c)\right)$ with vertices of $\Delta^{*}(a, b, c)$ given by

$$
\begin{gathered}
\nu_{0}^{*}=(0,0,0,0), \nu_{1}^{*}=(1,0,0,0), \nu_{2}^{*}=(0,1,0,0), \\
\nu_{3}^{*}=(-a,-b, 0,0), \nu_{4}^{*}=(0,0,0,1), \\
\nu_{5}^{*}=(-3 a,-3 b,-1,-1), \nu_{6}^{*}=(0,0,1,0), \nu_{7}^{*}=(-2 a,-2 b,-1,0) .
\end{gathered}
$$

The polar duality of the polytope determines uniquely the polytope $\Delta(a, b, c)$. Given the polytopes we may determine the Hodge numbers of the Calabi-Yau hypersurfaces to be $\left(h^{1,1}\left(X_{\Delta}\right), h^{2,1}\left(X_{\Delta}\right)\right)=$ $(3,243),(4,148),(5,101)$ for $\tilde{X}_{E(8)}, \tilde{X}_{E(7)}$ and $\tilde{X}_{E(6)}$, respectively. Using the formula (2.2) for the Chow ring, we see that $H^{1,1}\left(X_{\Delta}, \mathbf{Q}\right)$ has dimensions three for all $\tilde{X}_{E(k)}(k=6,7,8)$. The fact that we have less dimensions than those expected from $h^{1,1}\left(X_{\Delta}\right)$ for $\tilde{X}_{E(7)}$ and $\tilde{X}_{E(6)}$ means we have so-called twisted sectors for these models. There is a way to remedy this situation modifying the toric data slightly, however this is not important for our local mirror calculations.

For the dual of the Kähler cone in $H^{1,1}\left(X_{\Delta}, \mathbf{Q}\right)$, the Mori cone, we will find three generators (see [21] for detailed calculations):

$$
\begin{aligned}
& l^{(1)}=(0 ; 0,0,1,-1,-1,0,1), \\
& l^{(2)}=(0 ; 0,0,-3,1,1,1,0), \\
& l^{(3)}=(-d ; a, b, 0, c, 1,0,-1) .
\end{aligned}
$$

These bases have their duals $J_{1}, J_{2}$ and $J_{3}$, which are related to the toric divisors $D_{i}$ in (2.2) by

$$
D_{7}=J_{1}-J_{3}, D_{3}=J_{1}-3 J_{2}, D_{0}=-d J_{3},
$$

where $D_{0}=-\left(D_{1}+\cdots+D_{7}\right)$, the canonical class $K_{\tilde{\mathbf{P}}_{\Sigma(a, b, c)}}$. The ring 
structure of $H_{\text {toric }}^{\text {even }}\left(X_{\Delta}, \mathrm{Q}\right)$ may be recovered from the following data;

$$
\begin{array}{lll}
\tilde{X}_{E(6)}: 27 D_{H}^{3}+27 D_{3}^{3}+3 D_{7}^{3}, & \left(c_{2}, \vec{D}\right)=(90,-18,6) \\
\tilde{X}_{E(7)}: 18 D_{H}^{3}+18 D_{3}^{3}+2 D_{7}^{3}, & \left(c_{2}, \vec{D}\right)=(96,-12,8) \\
\tilde{X}_{E(8)}: 9 D_{H}^{3}+9 D_{3}^{3}+D_{7}^{3}, & \left(c_{2}, \vec{D}\right)=(102,-6,10)
\end{array}
$$

where we have used the convention that the coefficient of $D_{i} D_{j} D_{k}$ represents the corresponding cubic intersection number $\int_{X_{\Delta}} D_{i} D_{j} D_{k}$, and $\left(c_{2}, \vec{D}\right)=\left(\left(c_{2}, D_{H}\right),\left(c_{2}, D_{3}\right),\left(c_{2}, D_{7}\right)\right)$ with $D_{H}:=J_{1}$.

The above simple form of intersection numbers comes from the fact that the divisors $D_{7}$ and $D_{3}$ are isomorphic, respectively, to the del Pezzo surfaces $B l_{k}(k=6,7,8)$ and $\mathbf{P}^{2}$, which may be contracted to a point. In fact we see in (4.6) that the rational curves $l^{(3)}$ and $l^{(2)}$ are contained, respectively, in the divisors $D_{7}$ and $D_{3}$. Thus $l^{(3)}$ and $l^{(2)}$ are the extremal rays for the respective contractions. In the next subsection we will study in detail the monodromy formula (3.23) for the contractions $l^{(3)}$ and $l^{(2)}$.

\subsection{Local limit of the monodromy - del Pezzo surfaces $\mathbf{P}^{2}, B l_{6}, B l_{7}, B l_{8}$}

Now we are ready to consider the monodromy problem under the local mirror symmetry limit to the del Pezzo surfaces. We will focus on the primitive contractions corresponding the extremal rays $l^{(2)}$ and $l^{(3)}$, explicit in our models $\tilde{X}_{E(k)}$.

Specifying a contraction, say $l^{(3)}$, we have a subvariety which is isomorphic to $\mathbf{P}^{1}$ in the compactification of the moduli space of the complex structures of $\tilde{X}_{E(k)}^{\vee}$. Namely the local coordinate of the $\mathbf{P}^{1}$ about the LCSL is given by $z=(-1)^{l_{0}^{(3)}} a^{l^{(3)}}$ and the $\mathbf{P}^{1}$ is located at $x=$ $y=0\left(x=(-1)^{l_{0}^{(1)}} a^{l^{(1)}}, y=(-1)^{l_{0}^{(2)}} a^{l^{(2)}}\right)$. This is exactly the situation in which we take the local mirror symmetry limit to del Pezzo surfaces [9]. If we take $l^{(2)}$, then we will come to the local mirror symmetry limit to $\mathbf{P}^{2}$. In both cases the general formula (3.23) due to Horja applies. The Picard-Fuchs equations $(\mathcal{L} w=0)$ which describe our local mirror symmetry limits also follow from the data of the extremal ray $l$, and 
they are given by,

$$
\begin{aligned}
\mathbf{P}^{2} & : \mathcal{L}=\left\{\theta_{y}^{2}+3 y\left(3 \theta_{y}+2\right)\left(3 \theta_{y}+1\right)\right\} \theta_{y} \\
B l_{6} & : \mathcal{L}=\left\{\theta_{z}^{2}+3 z\left(3 \theta_{z}+2\right)\left(3 \theta_{z}+1\right)\right\} \theta_{z} \\
B l_{7} & : \mathcal{L}=\left\{\theta_{z}^{2}+4 z\left(4 \theta_{z}+3\right)\left(4 \theta_{z}+1\right)\right\} \theta_{z} \\
B l_{8} & : \mathcal{L}=\left\{\theta_{z}^{2}+12 z\left(6 \theta_{z}+5\right)\left(6 \theta_{z}+1\right)\right\} \theta_{z}
\end{aligned}
$$

We see from the form of the differential operators, the monodromy of the solutions is reducible for all cases, which will be interpreted later.

Now let us look closely our hypergeometric series (3.5) under the local mirror symmetry limit to the del Pezzo surfaces $B l_{6}, B l_{7}, B l_{8}$. In the IIB side the local mirror symmetry limit means the limit $x, y \rightarrow 0$ in the hypergeometric series. From our definition of the hypergeometric series combined with the ring structure of $H_{\text {toric }}^{\text {even }}\left(X_{\Delta}, \mathbf{Q}\right)$, it is straightforward to obtain the following form of the limit;

$$
\begin{aligned}
& \left.w_{0}\left(\vec{x}, \frac{\vec{J}}{2 \pi i}\right) \bmod \operatorname{Ann} D_{7}\right|_{x, y \rightarrow 0} \\
& =\mathbf{1}+D_{7}\left(-\frac{1}{2 \pi i} \partial_{\rho_{3}} w_{0}(z)\right)+D_{7}^{2}\left(\frac{1}{2} \frac{1}{(2 \pi i)^{2}} \partial_{\rho_{3}}^{2} w_{0}(z)\right) \\
& \quad+D_{7}^{3}\left(-\frac{1}{6} \frac{1}{(2 \pi i)^{3}} \partial_{\rho_{3}}^{3} w_{0}(z)\right),
\end{aligned}
$$

where $A n n D_{7}=\left\{v \in H_{\text {toric }}^{\text {even }}\left(X_{\Delta}, \mathbf{Q}\right) \mid D_{7} \cdot v=0\right\} \otimes \mathbf{C}[\log x]\{x\}$. Since the first three terms should give the local solutions of the 3rd order differential equations (4.9), we arrange the above formal expansion to $w_{0}\left(z, \frac{J}{2 \pi i}\right)=\left(\mathbf{1}, D_{7}, D_{7}^{2}\right) . \Pi_{B, l o c a l}(x)$ neglecting the $D_{7}^{3}$ term. In case of $\mathbf{P}^{2}$ we expand the series (3.5) via the basis $\Pi^{A, l o c a l}=\left(1, D_{3}, D_{3}^{2}\right)$, and correspondingly we have $\Pi_{B, l o c a l}(y)={ }^{t}\left(1,-\frac{1}{3} \partial_{\rho_{2}} w_{0}, \frac{1}{18} \partial_{\rho_{2}}^{2} w_{0}\right)$.

In Table 3, we have listed the monodromy matrices for the hypergeometric series $\Pi_{B, \text { local }}$. Though the the evaluations of the monodromy is straightforward, we present, for reader's convenience, our definitions of the hypergeometric series about $z=\infty$ and its connection matrix $N$ to the series about $z=0$. We should note that the monodromy about $z=0, \infty$ is easy to be determined. For $B l_{k}$ we have defined the hypergeometric series about $z=\infty$ for $j=0,1$, in addition to the obvious 
one 1 , by

$$
\begin{aligned}
w_{j}^{\infty}(z) & =\sum_{k=1}^{d-1}\left(1-\alpha^{a k}\right)\left(1-\alpha^{b k}\right)\left(1-\alpha^{c k}\right) \alpha^{k j} \tilde{w}_{k}(z), \\
\tilde{w}_{k}(z) & =\frac{1}{d} \frac{1}{(2 \pi i)^{3}} \sum_{N=0}^{\infty} \frac{\Gamma\left(a N+\frac{a k}{d}\right) \Gamma\left(b N+\frac{b k}{d}\right) \Gamma\left(N+\frac{k}{d}\right)^{2}}{\Gamma(d N+k) \Gamma\left(1+N+\frac{k}{d}\right)}(-z)^{-N-\frac{k}{d}},
\end{aligned}
$$

with $\alpha$ being the primitive root of $\alpha^{d}=1(d=a+b+c)$. The connection matrix has determined by analytic continuation of the above series back to the region $z=0$. $\left(\mathbf{P}^{2}\right.$ has the same hypergeometric series about $z=\infty$ as $B l_{6}$, but differs about $z=0$ because the $l$ vector is different. This simply results in the difference in the connection matrix and also the monodromy matrices.)

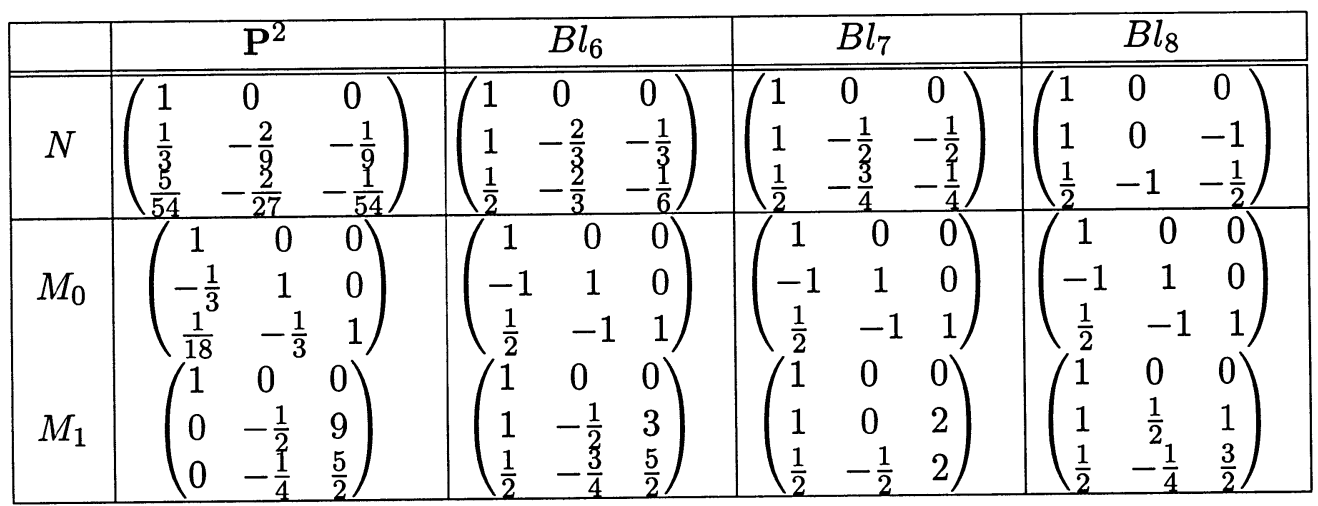

Table 3. The connection matrix and the monodromy matrices for del Pezzo surfaces. The connection matrix relates the hypergeometric series by $\Pi_{B, l o c a l}=N .{ }^{t}\left(1, w_{0}^{\infty}, w_{1}^{\infty}\right)$.

Corresponding to this hypergeometric series we consider the type IIA monodromy acting on the row vector $\Pi^{A, \text { local }}=\left(\mathbf{1}, D_{7}, D_{7}^{2}\right)$. Up to conjugation, the formula (3.23) for the type IIA monodromy may be identified with the monodromy about the discriminant dis $=1+$ $27 z, 1+64 z, 1+432 z$, for $B l_{6}, B l_{7}, B l_{8}$, respectively. In these cases the general formula (3.23) may be written

$$
\begin{aligned}
& \mathcal{T}_{3}: \gamma \mapsto \gamma-\left(1-\mathrm{e}^{D_{7}}\right) \\
& \cdot \int_{C_{\xi}} \frac{\left(1-\mathrm{e}^{-d \xi}\right)}{\left(1-\mathrm{e}^{-a \xi}\right)\left(1-\mathrm{e}^{-b \xi}\right)\left(1-\mathrm{e}^{\frac{2 D_{H_{H}+D_{6}}}{3}-\xi}\right)^{2}} \gamma(\xi) \frac{d \xi}{2 \pi i},
\end{aligned}
$$


where the residue is taken about $\xi=0$ and $\xi=\frac{2 D_{H}+D_{6}}{3}$. When writing this formula we use the rational equivalences, $D_{3}=a J_{3}, D_{2}=b J_{3}, D_{4}=$ $D_{5}=-J_{1}+J_{2}+J_{3}=-\frac{2 D_{H}+D_{6}}{3}+J_{3}$ and $D_{0}=-d J_{3}$. Also we use $D_{7}=D_{H}-J_{3}$ and the intersection numbers (4.8) when evaluating $\gamma(\xi)$ for $\gamma=1, D_{7}, D_{7}^{2}$. For the $\mathbf{P}^{2}$ contraction the corresponding formula is more simple and has the following form,

$$
\mathcal{T}_{2}: \gamma \mapsto \gamma-\left(1-\mathrm{e}^{D_{3}}\right) \int_{C_{\xi}} \frac{1}{\left(1-\mathrm{e}^{-\xi}\right)^{3}} \gamma(\xi) \frac{d \xi}{2 \pi i}
$$

Here we use $D_{3}=D_{H}-3 J_{2}$ for the determination $\gamma(\xi)$ for $\gamma=$ $1, D_{3}, D_{3}^{2}$.

In both cases it is easy to verify that the type IIA monodromy reproduces, up to conjugations, the monodromy $M_{1}$ about the discriminant for the hypergeometric series $\Pi_{B, l o c a l}$. Precisely for $B l_{k}(k=6,7,8)$ we verify that the monodromy operation $\mathcal{T}_{2}$ on the basis $\Pi^{A, l o c a l}$ exactly reproduces the matrix $M_{1}$ in Table 3 , and for $\mathbf{P}^{2}$ it coincides with $M_{0}^{-1} M_{1} M_{0}$ in Table 3. This is not merely a verification of the general results obtained in [17], but our point here is that we may arrange the expansion (4.10) using the pairing in "Theorem" 2. Namely we may arrange the hypergeometric series, for example, into the following,

$$
\begin{aligned}
& \left.w_{0}\left(\vec{x}, \frac{\vec{J}}{2 \pi i}\right) \bmod \operatorname{Ann} D_{7}\right|_{x, y \rightarrow 0} \\
& =w_{0}(z) \mathbf{1}+w^{(1)}(z)\left(D_{7}-\frac{1}{12} c_{2}\left(X_{\Delta}\right) D_{7}-\frac{1}{2} D_{7}^{2}\right) \\
& \quad+w^{(2)}(z) \frac{1}{h} D_{7}^{2}+w^{(3)}(z)\left(-\frac{1}{h} D_{7}^{3}\right)
\end{aligned}
$$

where we set $h=1,2,3$, respectively, for $B l_{8}, B l_{7}, B l_{6}$, and also note that $w_{0}(z), w_{1}(z), w_{2}(z)$ are linear combinations of $1, \partial_{\rho_{3}} w_{0}(z), \partial_{\rho_{3}}^{2} w_{0}(z)$. In the arrangement above (4.13), we have evaluated the Chern character of the ideal sheaf $\mathcal{I}_{p}$ on the del Pezzo surface $i: B l_{k} \hookrightarrow X_{\Delta}$;

$$
\operatorname{ch}\left(i_{*} \mathcal{I}_{p}\right)=D_{7}-\frac{1}{2} D_{7}^{2}-\frac{1}{12} c_{2}(X) D_{7}
$$

using $c_{1}\left(\mathcal{I}_{p}\right)=0, \operatorname{ch}_{2}\left(\mathcal{I}_{p}\right)=-\operatorname{Vol}_{S}$ for the ideal sheaf and $c_{1}\left(D_{7}\right)=-D_{7}$ which follows from the adjunction formula for $0 \rightarrow T_{S} \rightarrow T_{X} \rightarrow N \rightarrow 0$ 
with $\left.c_{1}(N)\right|_{S}=D_{7}$ and $c_{1}(X)=0$. We also use $\frac{1}{12}\left(c_{1}(S)^{2}+c_{2}(S)\right)=$ $\mathrm{Vol}_{S}$ for rational surfaces $S,\left(\chi\left(S, \mathcal{O}_{S}\right)=1\right)$. As for the 3-rd term of $(4.13), w^{(2)} \times \frac{1}{h} D_{7}^{2}$, we identify this with some sheaf $\mathcal{E}$, tensored with a suitable line bundle on it, supported on the canonical divisor $-c_{1}\left(D_{7}\right)=D_{7}$ of the surface (see (3.15)). These two sheaves should correspond to some 3-cycles in the mirror $X^{\vee}$, which are not explicit in our argument (see the discussions in the next section for recent works on this). We remark that the reducibility of the monodromy is evident in our interpretation because the D-brane charge $\mathbf{1}$ is not local but $\mathbf{1}=\operatorname{ch}\left(\mathcal{O}_{X}\right)$.

As we see in Table 4, after arranging the expansion of the hypergeometric series to (4.13), the monodromy matrices becomes integral. This means that the sheaves we looked above may be part of the integral generators of $K_{\text {hol }}(X)$. In case of $\mathbf{P}^{2}$, however, the monodromy matrix contains one rational number $\frac{1}{3}$. This should be understood by the fact that the anti-canonical class $c_{1}\left(D_{3}\right)$ of $\mathbf{P}^{2}$ is not a primitive class but three times of the line on it.

\begin{tabular}{|c|c|c|c|c|c|c|}
\hline & $\mathbf{P}^{2}$ & $B l_{6}$ & $B l_{7}$ & \multicolumn{2}{|c|}{$B l_{8}$} \\
\hline \hline \multirow{3}{*}{$M_{0}$} & $\left(\begin{array}{ccc}1 & 0 & 0 \\
-\frac{1}{3} & 1 & 0 \\
-1 & -3 & 1\end{array}\right)$ & $\left(\begin{array}{ccc}1 & 0 & 0 \\
-1 & 1 & 0 \\
0 & -3 & 1\end{array}\right)$ & $\left(\begin{array}{ccc}1 & 0 & 0 \\
-1 & 1 & 0 \\
0 & -2 & 1\end{array}\right)$ & $\left(\begin{array}{ccc}1 & 0 & 0 \\
-1 & 1 & 0 \\
0 & -1 & 1\end{array}\right)$ \\
$M_{1}$ & $\left(\begin{array}{ccc}1 & 0 & 0 \\
0 & -5 & 1 \\
0 & -36 & 7\end{array}\right)$ & $\left(\begin{array}{ccc}1 & 0 & 0 \\
1 & -2 & 1 \\
3 & -9 & 4\end{array}\right)$ & $\left(\begin{array}{ccc}1 & 0 & 0 \\
1 & -1 & 1 \\
2 & -4 & 3\end{array}\right)$ & $\left(\begin{array}{ccc}1 & 0 & 0 \\
1 & 0 & 1 \\
1 & -1 & 2\end{array}\right)$ \\
\hline
\end{tabular}

Table 4. Integral monodromy matrices for $S=B l_{k}(k=$ $6,7,8)$. We identify the corresponding sheaves with $\mathcal{O}_{X}, i_{*} \mathcal{I}_{p}, i_{*} \mathcal{E}$ in $D(X)$. Monodromy matrices of $\mathbf{P}^{2}$ and $B l_{k}$ have also been calculated, respectively, in [13] and [31].

\section{Discussions}

We have proposed the monodromy invariant pairing (3.10) between the D-brane charges. As we have argued, this pairing has its ground on the homological mirror symmetry due to Kontsevich. It is interesting to note that the pairing was essentially utilized when evaluating 
the prepotential in the very beginning of the mirror symmetry and its application to Gromov-Witten invariants.

In the following we summarize related subjects which we haven't addressed in this article.

In this paper we have restricted our attentions to the primitive contractions, where the extremal rays or equivalently some edges in the secondary polytope play their roles to specify the torus invariant orbit $\mathbf{P}^{1}$ in the complex structure moduli space. Zariski theorem of Lefschetz type for hypersurfaces says that for a generic line $\mathbf{P}^{1}$, generic to the discriminant variety, there is a surjective homomorphism $\pi_{1}\left(\mathrm{P}^{1} \backslash\right.$ $\left(\right.$ Dis $\left.\left.\cap \mathbf{P}^{1}\right)\right) \rightarrow \pi_{1}(\overline{\mathcal{M}} \backslash D i s)$ for the deformation space $\overline{\mathcal{M}}$. It is not clear the analysis restricting to the torus invariant $\mathbf{P}^{1}$ suffices to find all necessary generators for the monodromy group, although for the one, two parameter examples we looked in this paper we verify that the monodromy group is generated by them.

The Calabi-Yau hypersurfaces we have looked in the last section admit topology changes due to the flop operations. According to [1] the (complexified) Kähler moduli spaces of topologically different CalabiYau manifolds $X$ and $X^{\prime}$ are unified in the complex structure moduli space of their mirror $X^{\vee}$ by analytic continuation. Combined with the homological mirror symmetry, we should have the categorical equivalence $D(X) \cong D\left(X^{\prime}\right)$ for their derived categories. The full picture of the moduli spaces of our models in this respect will be reported elsewhere.

Finally we address to recent progresses in physics. The homological mirror symmetry due to Kontsevich may be interpreted as the mirror symmetry of D-branes in string theory. In ref. [40] the conformal field theory analogue (boundary states) of the D-branes was first formulated, and more recently in the work [5] the relations between the geometry of the cycles and the boundary states of conformal filed theory (at Geppnar point) have been pursued. In this respect the two parameter models we looked in the text have also been analyzed in refs. [11], [24], [41]. The local mirror symmetry limit of these D-brane analysis considered in [13]. Especially the local mirror symmetry limit and also the monodromy problem have been studied in general using LandauGinzburg theory in a recent paper [16], which appeared when this article was being completed. The paper [16] proposes a way to construct 3-cycles which corresponds to sheaves on (toric) del Pezzo surfaces, 
which we haven't looked in this paper. Our pairing contained in the formal expansion of the hypergeometric series (3.5), (3.6) provides us the corresponding hypergeometric series to a sheaf without specifying the 3-cycle explicitly, and seems to provide a complementary approach to [16]. We will come to this problem in future investigations.

\section{A Brief summary of the monodromy cal- culations}

In this appendix, following [6], we briefly summarize the monodromy calculations done in the text. Here we write the case of the quartic hypersurface in $\mathbf{P}^{3}$, the extension to other cases are straightforward. As described in section 2, the relevant hypergeometric series about a LCSL are determined by the toric data $l=(-4 ; 1,1,1,1)$, from which we have $w_{0}(x)=\sum_{n=0}^{\infty} \frac{(4 n) !}{(n !)^{4}} x^{n}$. The analytic continuation to $x=\infty$ may be done by the Barnes integral representation

$$
\begin{aligned}
w_{0}(x) & =\sum_{n=0}^{\infty} \frac{(4 n) !}{(n !)^{4}} x^{n} \\
& =\frac{1}{2 \pi i} \int_{C} d s \Gamma(-s) \frac{\Gamma(4 s+1)(-1)^{s}}{\Gamma(s+1)^{3}} x^{s} \\
& =\frac{1}{2 \pi i} \int_{C} d s \Gamma(4 s+1) \frac{\Gamma(-s)(-1)^{s}}{\Gamma(s+1)^{3}} x^{s} \\
& =-\frac{1}{4} \sum_{m=1}^{\infty} \frac{\Gamma\left(\frac{m}{4}\right)}{\Gamma(m) \Gamma\left(1-\frac{m}{4}\right)^{3}}(-1)^{\frac{3 m}{4}} x^{-\frac{m}{4}},
\end{aligned}
$$

where in the second line the contour $C$ encircle the poles at $s=$ $0,1,2, \ldots$ counterclockwise, while in the third line it is deformed to encircle the poles at $s=-\frac{m}{4}(m=1,2, \ldots)$ clockwise. This completes the analytic continuation to $x=\infty$. Other hypergeometric series about $x=\infty$ are defined by

$$
w_{j}^{\infty}(x)=-\frac{1}{4} \sum_{m=1}^{\infty} \frac{\Gamma\left(\frac{m}{4}\right)}{\Gamma(m) \Gamma\left(1-\frac{m}{4}\right)^{3}} \alpha^{\frac{3 m}{2}} \alpha^{m j} x^{-\frac{m}{4}} \quad(j=0,1,2,3),
$$


where $\alpha$ is the primitive root of $\alpha^{4}=1$. Setting $m=4 N+k$ and using the relation $\Gamma(z) \Gamma(1-z)=\frac{\pi}{\sin \pi z}$, we may rewrite (A.2) as

$$
\begin{aligned}
w_{j}^{\infty}(x) & =\sum_{k=1}^{4}\left(1-\alpha^{k}\right) \alpha^{k j} \tilde{w}_{k}(x), \\
\tilde{w}_{k}(x) & =\frac{1}{4} \frac{1}{(2 \pi i)^{3}} \sum_{N=0}^{\infty} \frac{\Gamma\left(N+\frac{k}{4}\right)^{4}}{\Gamma(4 N+k)} x^{-N-\frac{k}{4}} .
\end{aligned}
$$

These four series are not independent but have one relation $w_{0}^{\infty}+w_{1}^{\infty}+$ $w_{2}^{\infty}+w_{3}^{\infty}=0$. For the analytic continuation back to $x=0$ we utilize again the following integral representation for $\tilde{w}_{k}(x)$;

$$
\begin{aligned}
\tilde{w}_{k}(x) & =\int_{C_{1}} \frac{d s}{\mathrm{e}^{2 \pi i}-1} \frac{1}{4} \frac{1}{(2 \pi i)^{3}} \frac{\Gamma\left(s+\frac{k}{4}\right)^{4}}{\Gamma(4 s+k)} x^{-s-\frac{k}{4}} \\
& =-\int_{C_{1}^{\prime}} \frac{d s}{\mathrm{e}^{2 \pi i}-1} \frac{1}{4} \frac{1}{(2 \pi i)^{3}} \frac{\Gamma\left(s+\frac{k}{4}\right)^{4}}{\Gamma(4 s+k)} x^{-s-\frac{k}{4}},
\end{aligned}
$$

where in the first line $C_{1}$ encircles the poles at $s=0,1,2, \ldots$ counterclockwise, while in the second line it is deformed to $C_{1}^{\prime}$ which encircles the higher 3 rd order poles at $s=-m-\frac{k}{4}(m=0,1,2, \ldots)$ counterclockwise. The higher order poles above introduce the logarithms about $x=0$, up to $(\log x)^{2}$, which should be connected to the logarithmic singularity in $w_{0}, \partial_{\rho} w_{0}, \partial_{\rho}^{2} w_{0}$ at our LCSL. The connection matrix may be determined simply by comparing our solutions at LCSL and the analytic continuation of $w_{j}^{\infty}(x)$. The monodromy at LCSL simply comes from the logarithms in the hypergeometric series, and easily determined. Also the monodromy about $x=\infty$ is easily determined. This is because the monodromy is represented in the basis $w_{j}^{\infty}(x)$ by $w_{0}^{\infty} \rightarrow w_{1}^{\infty}, w_{1}^{\infty} \rightarrow w_{2}^{\infty}, w_{2}^{\infty} \rightarrow w_{3}^{\infty}=-w_{0}^{\infty}-w_{1}^{\infty}-w_{2}^{\infty}$ using the relation noted above.

Monodromy matrices and the connection matrix appeared in the text are determined in this way.

\section{References}

[1] P.S. Aspinwall, B.R. Greene, and D.R. Morrison, Multiple mirror manifolds and topology change in string theory, Phys. Lett., B303 (1993), 249-259. 
[2] V.V. Batyrev, Dual polyhedra and mirror symmetry for Calabi-Yau hypersurfaces in toric varieties, J. Alg. Geom., 3 (1994), 493-535.

[3] A. Bondal and D. Orlov, Semiorthogonal decompositions for algebraic varieties, alg-geom/9506012.

[4] T. Bridgeland, Equivalences of triangulated categories and FourierMulai transforms, Bull. London Math. Society, 31 (1999), 25-34, math. AG/9809114.

[5] I. Brunner, M.R. Douglas, A. Lawrence, and C. Römelsberger, D-branes on the Quintic, hep-th/9906200.

[6] P. Candelas, X.C. de la Ossa, P.S. Green, and L.Parkes, A pair of Calabi-Yau manifolds as an exactly soluble superconformal theory, Nucl.Phys., B356 (1991), 21-74.

[7] P. Candelas, X.C. de la Ossa, A. Font, S. Katz and D.R. Morrison, Mirror symmetry for two parameter models - I, Nucl. Phys., B416 (1994), 481-538, hep-th/9308083.

[8] P. Candelas, X.C. de la Ossa, A. Font, S. Katz and D.R. Morrison, Mirror symmetry for two parameter models - II, Nucl. Phys., B429 (1994), 626-674, hep-th/9403187.

[9] T.-T. Chiang, A. Klemm, S.-T. Yau, and E. Zaslow, Local Mirror Symmetry: Calculations and Interpretations, hep-th/9903053.

[10] D. Cox, The homogeneous coordinate ring of a toric variety, J. Alg. Geom., 4 (1995), 17-50, alg-geom/9210008.

[11] D. Diaconescu and C. Römelsberger, D-branes and bundles on elliptic fibrations, hep-th/9910172.

[12] I.V. Dolgachev, Mirror symmetry for lattice polarized K3 surfaces, Algebraic geometry, 4; J. Math. Sci., 81(3) (1996), 2599-2630.

[13] M. Douglas, B. Fiol, and C. Römelsberger, The spectrum of BPS branes on a noncompact Calabi-Yau, hep-th/0003263.

[14] W. Fulton, Introduction to Toric Varieties, Ann. of Math. Studies, 131, Princeton University Press, Princeton, New Jersey, 1993. 
[15] A.B. Givental, Equivariant Gromov-Witten invariants, Internat. Math. Res. Notices, 13 (1996), 613-663.

[16] K. Hori, A. Iqbal; and C. Vafa, D-branes and mirror symmetry, hep-th/0005247.

[17] R.P. Horja, Hypergeometric Functions and Mirror Symmetry in Toric Varieties, math.AG/9912109.

[18] S. Hosono, GKZ systems, Gröbner fans and moduli spaces of Calabi-Yau hypersurfaces in "Topological Field Theory, Primitive Forms and Related Topics" Progress in Mathematics, Birkhäuser, pp. 239-265, alg-geom/9707003.

[19] S. Hosono, A. Klemm, S. Theisen, and S.-T. Yau, Mirror symmetry, mirror map and applications to Calabi-Yau hypersurfaces, Commun. Math. Phys., 167 (1995), 301-350, hep-th/9308122.

[20] S. Hosono, A. Klemm, S. Theisen, and S.-T. Yau, Mirror symmetry, mirror map and applications to complete intersection CalabiYau spaces, Nucl. Phys., B433 (1995), 501-554, hep-th/9406055.

[21] S. Hosono, B.H. Lian, and S.-T. Yau, GKZ-Generalized hypergeometric systems in mirror symmetry of Calabi-Yau hypersurfaces, Commun. Math. Phys., 182 (1996), 535-577, alg-geom/9511001.

[22] S. Hosono, B.H. Lian, and S.-T. Yau, Maximal degeneracy points of GKZ systems, J. Amer. Math. Soc., 10 (1997), 427-443, alg-geom/9603014.

[23] S. Hosono, B.H. Lian, and S.-T. Yau, Type IIA monodromy of Calabi-Yau manifolds, 1997 (unpublished).

[24] P. Kaste, W. Lerche, C.A. Lütken and J. Walcher, D-branes on K3-fibrations, hep-th/9912147.

[25] A. Klemm, W. Lerche, and P. Mayr, K3 fibrations and heterotic type II string duality, Phys. Lett., B357 (1995), 313-322, hep-th/9506112.

[26] A. Klemm, P. Mayr, and C. Vafa, BPS States of Exceptional Non-Critical Strings, in the proceedings of the conference "Advanced Quantum Field Theory" (in memory of Claude Itzykson), hep-th/9607139. 
[27] A.Klemm and S.Theisen, Considerations of one modulus CalabiYau Compactifications: Picard-Fuchs equations, Kähler potentials and mirror maps, Nucl. Phys., B389 (1993), 153-180.

[28] M. Kobayashi, Duality of weights, mirror symmetry and Arnold's strange duality, alg-geom/9502004.

[29] M. Kontsevich, Homological algebra of mirror symmetry, in Proceedings of the International Congress of Mathematicians, Vol. 1, 2 (Züich, 1994), 120-139, Birkhäuser, Basel, 1995.

[30] M. Kontsevich, lectures, 1996 (unpublished).

[31] W. Lerche, P. Mayr, and N.P. Warner, Non-critical strings, del Pezzo singularities and Seiberg-Witten curves, Nucl.Phys., B499 (1997), 125-148, hep-th/9612085.

[32] B.H. Lian, K. Liu, and S.-T. Yau, Mirror principle I, II, Asian J. Math., 1(4) (1997), 729-763, and Asian J. Math., 3(1) (1999), 109-146, alg-geom/9712011, math/9905006.

[33] B.H. Lian and S.-T. Yau, Arithmetic properties of the mirror map and quantum coupling, Commun. Math. Phys., 176 (1996), 163192 , hep-th/9411234.

[34] D.R. Morrison, Picard-Fuchs equations and mirror maps for hypersurfaces in "Essays on Mirror Manifolds" ed. S.-T.Yau, International Press, Hong Kong 1992, alg-geom/9202026.

[35] D.R. Morrison and C. Vafa, Compactifications of F-Theory on Calabi-Yau Threefolds - I, Nucl. Phys., B473 (1996), 74-92.

[36] D.R. Morrison and C. Vafa, Compactifications of F-Theory on Calabi-Yau Threefolds - II, Nucl. Phys., B476 (1996), 437-469.

[37] S. Mukai, Duality between $D(X)$ and $D(\tilde{X})$ with its application to Picard sheaves, Nagoya Math. J., 81, 153-175.

[38] S. Mukai, On the moduli spaces of bundles on a K3 surface I, Vector bundles on algebraic varieties (Bombay, 1984), Tata. Inst. Fund. Res., Bombay, (1987), 341-413. 
[39] T. Oda, Convex bodies and Algebraic Geometry, An Introduction to the Theory of Toric Varieties, A Series of Modern Surveys in Mathematics, Springer-Verlarg New York, 1985.

[40] H. Ooguri, Y. Oz, and Z. Yin, D-branes on Calabi-Yau spaces and their mirrors, Nucl.Phys., B477 (1996), 407-430, hep-th/9606112.

[41] E. Scheidegger, D-branes on one- and two-parameter Calabi-Yau hypersurfaces, JHEP, 0004 (2000), 003, hep-th/9912188.

[42] J. Stienstra, Resonant hypergeometric systems and mirror symmetry, in "Integrable systems and algebraic geometry" (Kobe/Kyoto 1997), World Sci. Publishing, River Edge, NJ, (1998), 412-452, alg-geom/9711002.

[43] A. Strominger, Special Geometry, Commun. Math. Phys., 133 (1990), 163-180.

[44] A. Strominger, S.-T. Yau, and E. Zaslow, Mirror symmetry is TDuality, Nucl. Phys., B479 (1996), 243-259.

[45] C. Vafa, Extending Mirror Conjecture to Calabi-Yau with Bundles, hep-th/9804131. 